\title{
Stability and morphology of cerium oxide surfaces in an oxidizing environment: A first-principles investigation
}

\author{
Marco Fronzi, ${ }^{1,2, a)}$ Aloysius Soon, ${ }^{1}$ Bernard Delley, ${ }^{3}$ Enrico Traversa, ${ }^{2}$ and \\ Catherine Stampfl ${ }^{1}$ \\ ${ }^{1}$ School of Physics, The University of Sydney, Sydney, New South Wales 2006, Australia \\ ${ }^{2}$ Department of Chemical Science and Technology, University of Tor Vergata, 00133 Rome, Italy \\ ${ }^{3}$ Paul-Scherrer-Institut, CH-5232 Villigen PSI, Switzerland
}

(Received 3 November 2008; accepted 7 July 2009; published online 8 September 2009)

\begin{abstract}
We present density functional theory investigations of the bulk properties of cerium oxides $\left(\mathrm{CeO}_{2}\right.$ and $\mathrm{Ce}_{2} \mathrm{O}_{3}$ ) and the three low index surfaces of $\mathrm{CeO}_{2}$, namely, (100), (110), and (111). For the surfaces, we consider various terminations including surface defects. Using the approach of "ab initio atomistic thermodynamics," we find that the most stable surface structure considered is the stoichiometric (111) surface under "oxygen-rich" conditions, while for a more reducing environment, the same (111) surface, but with subsurface oxygen vacancies, is found to be the most stable one, and for a highly reducing environment, the (111) Ce-terminated surface becomes energetically favored. Interestingly, this latter surface exhibits a significant reconstruction in that it becomes oxygen terminated and the upper layers resemble the $\mathrm{Ce}_{2} \mathrm{O}_{3}(0001)$ surface. This structure could represent a precursor to the phase transition of $\mathrm{CeO}_{2}$ to $\mathrm{Ce}_{2} \mathrm{O}_{3}$. (C) 2009 American Institute of Physics. [doi:10.1063/1.3191784]
\end{abstract}

\section{INTRODUCTION}

The study of the catalytic properties of materials plays a critical role in the development of intermediate temperature solid oxide fuel cell technologies. Cerium oxide, due to its peculiar and desirable characteristics, has been the object of intense interest for various heterogeneous catalytic reactions, including several key applications in solid oxide fuel cells. ${ }^{1,2}$ Because it behaves as a good ionic conductor, ${ }^{3,4}$ and at the same time as a good catalyst support for active metal nanoparticles, ${ }^{5,6}$ cerium oxide finds application both as an electrolyte and as an anode support. In anodic reactions, it plays an active part in the catalysis of the fuel cell, and thus it is crucial to investigate the chemical and physical properties of the oxide surfaces when exposed to an anodic fuel cell gas environment that can be pure fuel, in conventional cells, or can be, in a particular subclass of fuel cells, the single chamber fuel cells, a mixture of oxygen and fuel. ${ }^{7-9}$ For this reason it is therefore important to first understand the behavior of cerium oxide surfaces under different oxidizing conditions.

It should be mentioned that the electronic structure of materials that exhibit strong electron correlation, such as cerium oxide and many rare-earth oxides, is typically poorly described by the conventional density functional theory (DFT) approach. DFT averages the exchange correlation of the electronic interaction and does not correct for the unphysical self-interaction of the electrons. For the case of $\mathrm{CeO}_{2}$ and $\mathrm{Ce}_{2} \mathrm{O}_{3}$, the Ce- $4 f$ band is strongly localized, where for $\mathrm{CeO}_{2}$, the $\mathrm{Ce}-4 f$ band is unoccupied, thus strong correlation effects are absent. On the other hand, for $\mathrm{Ce}_{2} \mathrm{O}_{3}$ the

\footnotetext{
a) Author to whom correspondence should be addressed. Electronic mail: marco@physics.usyd.edu.au.
}

Ce- $4 f$ band is occupied and thus DFT is unable to provide a correct description of its electronic structure. In the latter case, DFT predicts a metallic behavior while, experimentally, $\mathrm{Ce}_{2} \mathrm{O}_{3}$ is known to be an antiferromagnetic semiconductor with a band gap of $2.40 \mathrm{eV} .{ }^{10}$ Recent studies have obtained an improved description of the electron structure and, in particular, of the band gap using a Hubbard term $U$ (Refs. 11-13) as a correction to the energy from DFT calculation.

In view of the importance of this class of material, both fundamentally and technologically, there has been a vast literature reported for both theoretical ${ }^{14-22}$ and experimental $^{23-30}$ studies on ceria and its surfaces. To date, both theoretical and experimental results obtained from these studies are still far from conclusive and are still actively debated. We first provide a brief overview of theoretical studies that have been performed for cerium oxide. For a more complete review, we refer the reader to Refs. 14 and 22 and references therein.

One of the early studies of $\mathrm{CeO}_{2}$ surfaces was performed for the (111) and (110) surfaces using simple interatomic potentials. ${ }^{31}$ It was found that the (111) surface is more stable than the (110) surface. This empirical approach was extended to other surfaces including higher index orientations such as (210) and (211). ${ }^{32}$ From that work, it was concluded that the (111) surface was by far the most stable, as found for most fluorite structures. Subsequently, $a b$ initio calculations were performed using the Hartree-Fock method. ${ }^{33,34}$ Similarly, the (111) surface was again predicted to be the most stable one, but with this approach a slightly better agreement with experimental bulk properties such as the lattice constant, the elastic constants, and the bulk modulus was obtained. Extending that work for the bulk properties of $\mathrm{CeO}_{2}$, Voloshina et $a l^{21}$ combined periodic Hartree-Fock calculations with 
corresponding wavefunction-based correlation finite-cluster calculations, i.e., employing the so-called method of correlation increment. With this correlation approach, they were able to explicitly include correlation effects (which can be important for such $f$-systems) and obtained a cohesive energy in excellent agreement with the experimental value. The calculated lattice constant and bulk modulus were also very close to the experimental values.

Turning to more recent DFT calculations of the defectfree surfaces of ceria, ${ }^{35-37}$ both the surface energies and electronic structure of the low-indexed surfaces of $\mathrm{CeO}_{2}$ have been studied using the projector augmented-wave (PAW) method with the local density approximation (LDA) and generalized gradient approximation (GGA). The work of Skorodumova $\mathrm{et} \mathrm{al.}{ }^{36}$ included results obtained with molecular dynamics simulations at $10 \mathrm{~K}$. In accord, out of all three low-index surfaces, namely, (100), (110), and (111), the (111) surface was found to be the most stable, agreeing well with experiment and previous calculations. The calculated surface energies were found to be $0.062 \mathrm{eV}^{-2}$ (LDA) and $0.044 \mathrm{eV} \AA^{-2}$ (GGA).$^{36}$ Using the same approach, a value of $0.039 \mathrm{eV}^{-2}$ was reported in Ref. 37 using the GGA. However, at this level of theory, the reported electronic structure of these surfaces suffers from the well-known intrinsic underestimation of the band gap.

To investigate the influence of strong electron correlation effects of the Ce- $4 f$ states and to investigate the degree of the localization in substoichiometric ceria and its surfaces, there have been a number of investigation employing approaches that go beyond standard DFT-GGA (DFT $+U$ and hybrid functionals). ${ }^{14-22}$ The most commonly employed approach is to include an empirical Hubbard $U$ term to artificially localize the $4 f$ electrons, ${ }^{15,17,19}$ commonly termed DFT $+U$. An alternative method employed is to include a controlled amount of Hartree-Fock exchange in the DFT framework, i.e., using the commonly known hybrid functionals. ${ }^{16,20,22}$ These approaches collectively improved the electronic structure description for these substoichiometric oxides of cerium, even reproducing the intrinsic band gap of defect-free $\mathrm{CeO}_{2}$ and $\mathrm{Ce}_{2} \mathrm{O}_{3}$. Specifically, one of the earliest studies involving the use of both DFT and DFT $+U$ was conducted by Jiang et al. ${ }^{38}$ They investigated the (111), (110), (210), (211), (100), and (310) surfaces of ceria with conventional DFT(LDA and GGA) and only the selected low-energy (111) surface with $\mathrm{LDA}+U$. The stability of the considered surfaces was evaluated as function of the oxygen partial pressure and temperature. Their study was confined to ideal surface terminations, without considering defect formation on those surfaces. At $300 \mathrm{~K}$, the stoichiometric (111) was found to have the lowest surface free energy for a wide range of oxygen partial pressures up to $1 \mathrm{~atm}$, while the Ce-terminated (111) surface becomes the most stable one only under ultrahigh vacuum conditions. For comparison, the authors chose the low-energy stoichiometric (111) surface and calculated its surface energy using $\mathrm{LDA}+U(U=7 \mathrm{eV})$, and the result obtained $(1.03 \mathrm{eV})$ was very close to that of LDA $(1.06 \mathrm{eV})$. This lead the authors to claim that the calculated surface energies may not show a strong dependence on the choice of the Hubbard $U$ term, and thus for this par- ticular work, the authors presented only the DFT-GGA calculated surface energies as a function of the oxygen pressure and temperature.

Concurrently, the atomistic and electronic structures of clean and reduced ceria surfaces were also studied by Fabris et al. ${ }^{30,39,40}$ The highlight of that work focused on and addressed the electron-localization effects responsible for the oxidation state change of cerium atoms when an oxygen vacancy is formed at the surface. The authors employed the $\mathrm{DFT}+U$ method, whereby the value of $U$ is determined from first principles, and the localized orbitals entering the Hubbard $U$ energy functional is also determined selfconsistently. That work also shows that the electronic and structural properties of the defect-free ceria surfaces calculated by $\mathrm{DFT}+U$ do not differ drastically from those calculated by conventional DFT. However, for reduced ceria surfaces (with oxygen vacancies), in order to describe more accurately the excess electrons, some kind of electron correlation treatment, such as the DFT $+U$ approach, was required. Oxygen vacancies were found to favor segregation to the (110) rather than to the (111) surface. However, one of the important conclusions of that work highlighted by the authors was that the relative energy of these reduced surface configurations considered in the work (i.e., involving both on- and subsurface vacancies) was highly dependent on (1) the DFT exchange-correlation functional approximation and (2) on the specific projectors used in the Hubbard $U$ term, in the DFT $+U$ approach. Even without considering the Hubbard term, the relative formation energy of these vacancies is also a subtle case, whereby DFT-LDA and DFT-GGA (with the $+U$ approach) give qualitatively different results. In particular, the former predicted that the on-surface vacancy was energetically favorable, while the latter predicted the subsurface $\mathrm{O}$ vacancy. Recent results have been reported by Ganduglia-Pirovano et al. ${ }^{41}$ where it was found using DFT with the Heyd-Scuseria-Ernzerhof (HSE06) hybrid functional, as well as the DFT $+U$ approach, that subsurface vacancies (below the first layer) with $(2 \times 2)$ periodicity are energetically more favorable by 0.45 (HSE06), 0.47 [PBE $+U$ (Perdew-Burke-Ernzerhof functional)], and $0.22 \mathrm{eV}$ $[\mathrm{LDA}+U]$, than on-surface oxygen vacancies. The same trend was reported by $\mathrm{Li}$ et al. ${ }^{42}$ Interestingly, a recent publication $^{43}$ reports that the values of $U$ presently used in the literature $(U>4 \mathrm{eV})$ tend to overestimate the binding energy of $\mathrm{CO}$ to ceria surfaces and that smaller values of $U=2-3 \mathrm{eV}$ seem to be more appropriate. Further, they found that the $\mathrm{CO}$ binding energy (and oxygen vacancy formation energy) is strongly dependent on the value of $U$. They also conclude that it is becoming evident that the values of $U$ presently used in the literature and giving the correct position of the filled gap state in reduced ceria $(U=4-5 \mathrm{eV})$, besides underestimating the reduction energy, can also yield a large overestimation of the $\mathrm{CO}$ binding energy to ceria surfaces.

From the discussion presented above, it is evident that it is still nontrivial to capture a satisfactory description of the Ce- $4 f$ electrons in these oxide systems, in particular, for surface properties such as the surface free energy. ${ }^{30,38-40}$ Even 
within the DFT $+U$ method, it is still arguably difficult to agree on a satisfactory way of specifying the value of the Hubbard term in this approach. ${ }^{15,19}$

In the present paper we extend the analysis of the surface stability, using DFT-GGA, to a large number of terminations of $\mathrm{CeO}_{2}$ both with and without surface defects. For each surface structure, we describe the atomic geometry, and for the more stable surfaces, we further analyze the electronic properties. To assess the relative stability of this large pool of surface structures, we employ the technique of $a b$ initio atomistic thermodynamics. This approach enables us to express the surface Gibbs free energies of any considered ceria surface in an oxygen environment. The effect of temperature and pressure is then included explicitly by taking into account its dependence on the oxygen chemical potential. In this way, we can obtain, as a first step, an understanding of the relative stability of "realistic" ceria surface structures, i.e. surfaces that are likely to exist under technical catalytic conditions.

It is well known that cerium oxides crystallize in two different structures that reflect the formal oxidation state of Ce: (1) the $\mathrm{CaF}_{2}$-fluorite structure for $\mathrm{CeO}_{2}$ where $\mathrm{Ce}$ is in the (IV) state and (2) the hexagonal structure for the sesquioxide, $\mathrm{Ce}_{2} \mathrm{O}_{3}$, where $\mathrm{Ce}$ is in the (III) state. ${ }^{35,44}$ As most of the interesting catalytic activities are reported for the fluorite phase, for this work, we will focus primarily on $\mathrm{CeO}_{2}$ and only briefly on $\mathrm{Ce}_{2} \mathrm{O}_{3}$. As the first step, using plain DFT, we study the structural and electronic ground-state properties of both bulk $\mathrm{CeO}_{2}$ and $\mathrm{Ce}_{2} \mathrm{O}_{3}$, i.e., the equilibrium lattice constant, bulk modulus, and heat of formation, and compare our results with experiment and with other available ab initio results. We then investigate various surface terminations of $\mathrm{CeO}_{2}$ for the low-index (100), (110), and (111) surfaces as well as the study of the $\mathrm{Ce}_{2} \mathrm{O}_{3}(0001)$ surface. These investigations include stoichiometric, as well as cerium- and oxygen-rich terminations, and structures containing surfaces defects in the first and second atomic layers. We proceed to calculate the surface Gibbs free energy as function of the oxygen chemical potential.

Having surveyed the current state-of-the-art calculations for these strongly electron correlated systems, it appears that there is still not a satisfactory approach to calculate the ground-state properties from first principles. In particular, we find that the influence of this strong electron correlation on relative energetics of ceria surfaces is still not well understood, although admittedly the electronic structure is clearly, but empirically, improved upon extending DFT to include the Hubbard $U$ term. Hence, the focus of this work is to provide a concise and detailed study of a large base of surface structures using DFT-GGA and compare this with available calculations going beyond plain DFT calculations (i.e., DFT $+U,{ }^{39}$ hybrid functionals, ${ }^{22}$ etc.) Using similar lowindex surface structures reported by Jiang et al. ${ }^{38}$ namely, the (100), (110), and (111) surfaces, and with the inclusion of surface defects (detailed later in the text), we find a good agreement in terms of the calculated DFT surface Gibbs free energies and thus the relative stability within the range of oxygen chemical potential considered, reproducing qualitative trends within the DFT-GGA framework.

\section{METHODOLOGY}

\section{A. DFT calculations: Basis set and convergence}

All calculations are performed using DFT and the GGA due to Perdew et al. ${ }^{45}$ for the exchange-correlation functional as implemented in the all-electron $\mathrm{DMol}^{3}$ code. The $\mathrm{DMol}^{3}$ method employs fast converging three-dimensional numerical integrations to calculate the matrix elements occurring in the Ritz variational method. The wave functions are expanded in terms of a double-numerical quality localized basis set with a real-space cutoff of 11 bohrs. Polarization functions and scalar-relativistic corrections are incorporated explicitly. More details of the $\mathrm{DMol}^{3}$ code can be found elsewhere. ${ }^{46,47}$

To simulate the surfaces we use supercells containing symmetric slabs. The thickness of the slabs correspond to 15 atomic layers for the (111) surfaces, 11 atomic layers for the (100), and 13 atomic layers for the (110) surfaces. The outermost atomic layers are fully relaxed for all surfaces; in particular, six layers for the (111) and two layers for both the (110) and the (100) surfaces. Various convergence tests are carried out to determine the optimal number of relaxed layers (see Appendix). A vacuum region between repeated slabs of about $30 \AA$ is created to ensure negligible interaction between adjacent slabs.

The Brillouin-zone (BZ) integration is performed, for bulk $\mathrm{CeO}_{2}$, using a Monkhorst-Pack (MP) grid of $8 \times 8 \times 8$, yielding $35 \mathbf{k}$-points in the irreducible part of the BZ (IBZ). For bulk $\mathrm{Ce}_{2} \mathrm{O}_{3}$, a $6 \times 6 \times 4$ k-point MP mesh was used resulting in $24 \mathbf{~ k - p o i n t s ~ i n ~ t h e ~ I B Z . ~ F o r ~ t h e ~ s u r f a c e ~ c a l c u l a - ~}$ tions, using $(2 \times 2)$ supercells, an $8 \times 8 \times 1 \mathbf{k}$-point mesh is used, yielding 16 and $10 \mathbf{k}$-points in the irreducible part of the IBZ for the (110) and (111) surfaces, respectively. For the (100) surface we use a $(\sqrt{2} \times \sqrt{2}) R 45^{\circ}$ supercell with an 8 $\times 8 \times 1 \mathbf{k}$-point mesh that yields ten $\mathbf{k}$-points in the irreducible part of the IBZ. With this basis set, the surface energies of different structures are converged to within $2 \mathrm{meV} / \AA^{2}$ with respect to k-points and the real-space cutoff parameter.

\section{RESULTS AND DISCUSSION}

\section{A. $A b$ initio atomistic thermodynamics}

In order to describe the thermodynamic stability of $\mathrm{CeO}_{2}$ surfaces in an oxygen environment, we use the DFT-derived total energies as input into an atomistic thermodynamics framework, ${ }^{48-51}$ which treats the effect of the surrounding gas phase as a corresponding reservoir in thermodynamic equilibrium with the surface. In equilibrium with such a reservoir, the most stable surface structure in the constant pressure and temperature $(p, T)$ ensemble minimizes the surface free energy, which is defined as

$$
\gamma\left(p_{i}, T\right)=\frac{1}{2 A}\left[G-\sum_{i} N_{i} \mu_{i}\left(p_{i}, T\right)\right] .
$$

Here $G$ is the Gibbs free energy of the solid with the surface area $A$, to which in a supercell calculation with symmetric slabs (as used in the present work) both the top and bottom contribute equally (hence the factor of $1 / 2) . \mu_{i}\left(p_{i}, T\right)$ is the pressure and temperature-dependent chemical potential of 


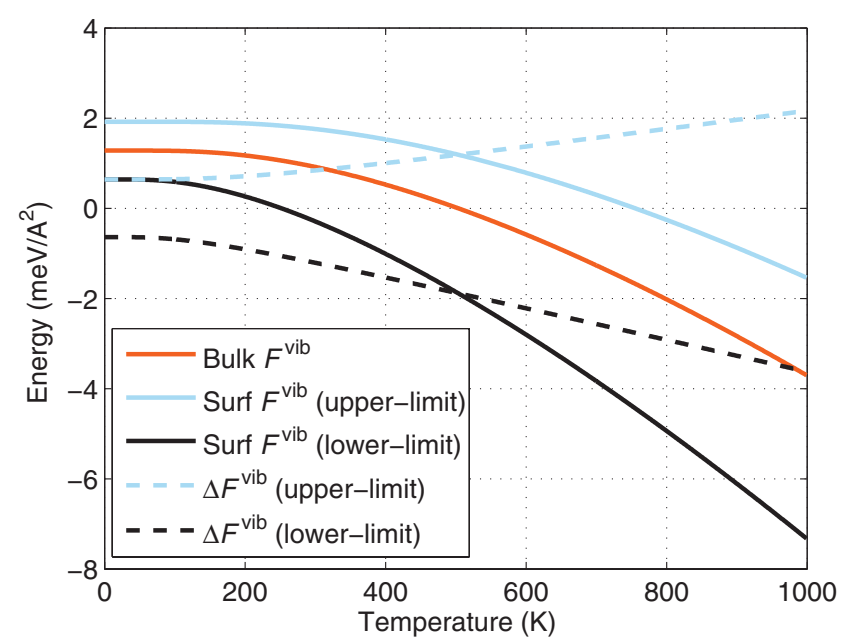

FIG. 1. Change in the vibrational contribution to the surface free energy, $\Delta F^{\mathrm{vib}}$. The solid midgray (red) line (bulk $F^{\mathrm{vib}}$ ) represents the vibrational contribution of the bulk to the total energy. The light (blue) and black lines represent the contribution of a generic surface estimated by varying the bulk modes by $+50 \%$ [surf $F^{\mathrm{vib}}$ (upper limit)] and $-50 \%$ [surf $F^{\mathrm{vib}}$ (lower limit)], respectively. The light (blue) and black dashed lines $\left[\Delta F^{\mathrm{vib}}\right.$ (upper limit) and $\Delta F^{\mathrm{vib}}$ (lower limit)] represent the difference between bulk $F^{\mathrm{vib}}$ and surf $F^{\mathrm{vib}}$ (upper limit) and surf $F^{\mathrm{vib}}$ (lower limit), respectively.

the various species $i$ present in the system, i.e., in this case $i=\mathrm{Ce}$ and O. $N_{i}$ is the total number of atoms in the component $i$ in the system. For ambient temperatures and sufficiently large particles, bulk $\mathrm{CeO}_{2}$ may further be assumed to be a second thermodynamic reservoir with which the surface is equilibrated. This constrains the chemical potentials of $\mathrm{Ce}$ and $\mathrm{O}$ to the Gibbs free energy of bulk $\mathrm{CeO}_{2}, g_{\mathrm{CeO}_{2}}^{\text {bulk }}$ (where the small $g$ denotes the Gibbs free energy per formula unit) and allows one to eliminate $\mu_{\mathrm{Ce}}$ from Eq. (1). The remaining quantities to be determined for the calculation of the surface free energy are then the oxygen chemical potential, as well as the difference in Gibbs free energies of the slab and bulk $\mathrm{CeO}_{2}$.

Determination of the oxygen chemical potential $\mu_{\mathrm{O}}$ is fixed by the surrounding gas phase reservoir, which may be approximated as an ideal gas. Ideal-gas laws then relate the chemical potential to pressure and temperature, ${ }^{48,50,52}$

$$
\mu_{\mathrm{O}}(p, T)=\frac{1}{2}\left[E_{\mathrm{O}_{2}}+\tilde{\mu}_{\mathrm{O}_{2}}\left(p^{0}, T\right)+k_{B} T \ln \left(\frac{p_{\mathrm{O}_{2}}}{p^{0}}\right)\right],
$$

where $T$ and $p$ represent the temperature and pressure and $p^{0}$ denotes atmospheric pressure. $\tilde{\mu}_{\mathrm{O}_{2}}\left(T, p^{0}\right)$ includes contributions from rotations and vibrations of the molecule, as well as the ideal-gas entropy at $1 \mathrm{~atm}$ which can be calculated or taken from experimental values listed in thermodynamic tables $^{53}$ as done in the present work. $k_{B}$ is the Boltzmann constant and $E_{\mathrm{O}_{2}}$ is the total energy of the oxygen molecule. The Gibbs free energy difference between the bulk phase and the slab [see Eq. (3)] has contributions from changes in the vibrational and configurational degrees of freedom at the surface, as well as from the expansion work term $(\Delta(p V))$, and as the main contribution, the difference in total energies $\left(\Delta E^{\mathrm{tot}}\right)$,

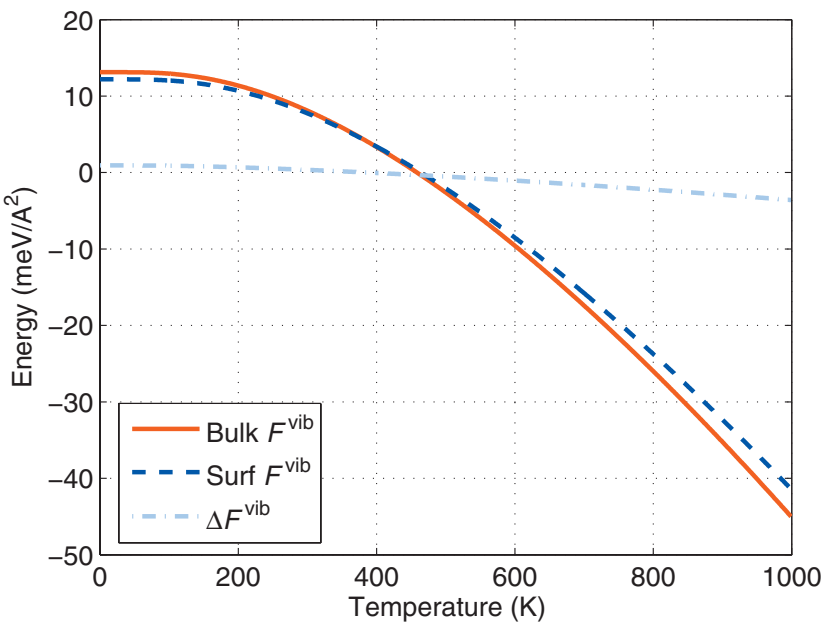

FIG. 2. Change in the vibrational contribution to the surface free energy, $\Delta F^{\mathrm{vib}}$, pale gray (pale blue) dot-dashed line. The solid (red) line (bulk $F^{\mathrm{vib}}$ ) represents the vibrational contribution of the bulk to the total energy and the

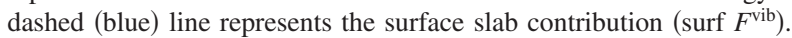

$$
\Delta G=\Delta E^{\mathrm{tot}}+\Delta F^{\mathrm{vib}}+\Delta F^{\mathrm{conf}}+\Delta(p V) .
$$

From dimensional analysis, the $\Delta(p V)$ term can be neglected. ${ }^{48}$ The configurational contribution $\left(\Delta F^{\text {conf }}\right)$ for a system such as $\mathrm{CeO}_{2}$ is neglected since we aim for a first comparison of the relative stability of different surface terminations. The vibrational contribution $\left(\Delta F^{\mathrm{vib}}\right)$ can be estimated from first principles using the computed phonon density of states of the surface and in the bulk. However, for simplicity, we apply the Einstein approximation ${ }^{54}$ to the phonon density of states and estimate the order of magnitude of this contribution,

$$
\begin{aligned}
\Delta F^{\mathrm{vib}}= & \frac{1}{A}\left[F^{\mathrm{vib}}\left(T, \bar{\omega}_{\mathrm{Ce}}^{\text {surf }}\right)-F^{\mathrm{vib}}\left(T, \bar{\omega}_{\mathrm{Ce}}^{\text {bulk }}\right)\right. \\
& \left.+2\left\{F^{\mathrm{vib}}\left(T, \bar{\omega}_{\mathrm{O}}^{\text {surf }}\right)-F^{\mathrm{vib}}\left(T, \bar{\omega}_{\mathrm{O}}^{\text {bulk }}\right)\right\}\right],
\end{aligned}
$$

where $\omega$ is the frequency of each vibrational mode and

$$
F^{\mathrm{vib}}(T, \omega)=\frac{1}{2} \hbar \omega+k_{B} T \ln \left(1-e^{-\hbar \omega / k_{B} T}\right) .
$$

$F^{\mathrm{vib}}\left(T, \bar{\omega}_{\mathrm{X}}^{\text {bulk }}\right)$ and $F^{\mathrm{vib}}\left(T, \bar{\omega}_{\mathrm{X}}^{\text {surf }}\right)$ are the absolute vibrational contributions to the surface free energy due to a bulk and a surface atom, respectively, with $\mathrm{X}$ being either $\mathrm{Ce}$ or $\mathrm{O}$.

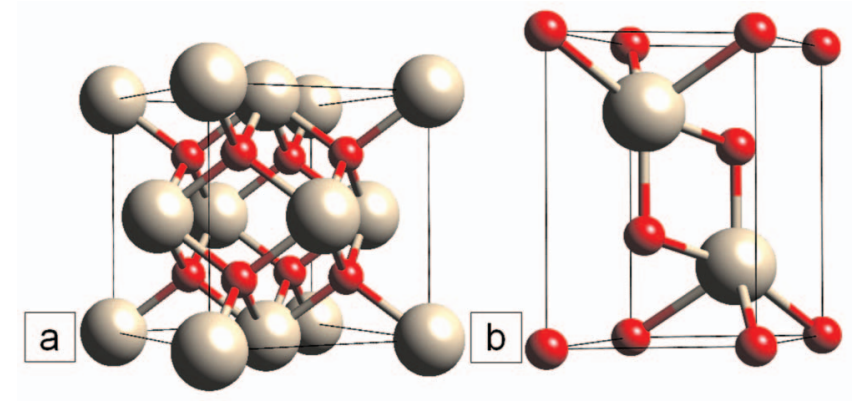

FIG. 3. The bulk unit cell of (a) $\mathrm{CeO}_{2}$ (fluorite structure) and (b) $\mathrm{Ce}_{2} \mathrm{O}_{3}$ (hexagonal structure). Small dark (red) spheres indicate oxygen atoms and large light gray sphere $\mathrm{Ce}$ atoms. This rendering is used in other figures hereafter. 
TABLE I. Bulk properties of $\mathrm{CeO}_{2}$ calculated using the LDA and GGA-PBE. $a(\AA), B_{0}(\mathrm{GPa}), E_{\mathrm{coh}}(\mathrm{eV})$, and $\Delta H_{f}(\mathrm{eV})$ refer to the bulk lattice constant, bulk modulus, cohesive energy, and heat of formation, respectively. The indirect $\mathrm{O}-2 p-\mathrm{Ce}-4 f$ band gap is represented by $E_{g}$ in $\mathrm{eV}$. Results of other first-principle calculations and experiment are listed for comparison. The full-potential augmented plane wave approach is denoted as FPLAPW. The Gaussian local-orbital method is abbreviated by GTO while PAW refers to the PAW method. The hybrid DFT functionals due to Heyd-Scuseria-Ernzerhof is referred to as DFT-HSE, (Ref. 66) and Adamo and Barone as PBE0 (Ref. 67), while the meta-GGA due to TPss Staroverov and Scuseria is referred to as TPSS (68).

\begin{tabular}{|c|c|c|c|c|c|}
\hline & $a$ & $B_{0}$ & $E_{\text {coh }}$ & $\Delta H_{f}$ & $E_{g}$ \\
\hline $\mathrm{DMol}^{3}(\mathrm{LDA})^{\mathrm{a}}$ & 5.36 & 222.7 & -25.59 & -11.06 & 2.11 \\
\hline a & 5.47 & 195.0 & -21.88 & -10.03 & 1.97 \\
\hline PAW-LDA ${ }^{\mathrm{b}}$ & 5.37 & 201.0 & -24.55 & -11.49 & 2.0 \\
\hline PAW-PBE ${ }^{\mathrm{b}}$ & 5.47 & 172.0 & -21.04 & -10.24 & 2.0 \\
\hline FPLAPW-PBE ${ }^{\mathrm{b}}$ & 5.47 & 170.0 & -21.15 & -10.35 & $\ldots$ \\
\hline $\mathrm{PAW}-\mathrm{LDA}+U^{\mathrm{b}}$ & 5.40 & 210.0 & $\cdots$ & $\cdots$ & 2.5 \\
\hline $\mathrm{PAW}-\mathrm{PBE}+U^{\mathrm{b}}$ & 5.49 & 180.0 & $\ldots$ & $\cdots$ & 2.25 \\
\hline PAW-PBE0 $0^{\mathrm{b}}$ & 5.39 & $\ldots$ & -19.13 & -11.15 & 4.5 \\
\hline PAW-HSE ${ }^{\mathrm{b}}$ & 5.40 & $\ldots$ & -19.70 & -11.00 & 3.5 \\
\hline GTO-LDA ${ }^{c}$ & 5.36 & 204.3 & $\ldots$ & $\ldots$ & 1.6 \\
\hline GTO-PBE ${ }^{\mathrm{c}}$ & 5.47 & 171.1 & $\cdots$ & $\cdots$ & 1.7 \\
\hline GTO-TPSS $^{\mathrm{c}}$ & 5.45 & 183.0 & $\cdots$ & $\cdots$ & 1.8 \\
\hline GTO-HSE $^{c}$ & 5.41 & 206.1 & $\ldots$ & $\ldots$ & 3.3 \\
\hline Expt. $^{\mathrm{d}}$ & 5.41 & 230.0 & $\ldots$ & -10.62 & 3.00 \\
\hline
\end{tabular}

We adopt the approach used in Ref. 48 and calculate the characteristic vibrational modes of $\mathrm{Ce}(30 \mathrm{meV})$ and $\mathrm{O}$ (49 meV) in bulk $\mathrm{CeO}_{2}$, taking the average of the vibrational modes due to each $\mathrm{Ce}$ and $\mathrm{O}$. This is done by performing a frozen-phonon calculation for each atom type in $\mathrm{CeO}_{2}$ at the $\Gamma$ point. Coupled modes between $\mathrm{Ce}$ and $\mathrm{O}$ are neglected. We define an upper and lower limit to the change in the vibrational contribution to the free energy by using a $50 \%$ variation of these bulk values to estimate the contribution of the surface $\mathrm{Ce}$ and $\mathrm{O}$ atoms. ${ }^{55}$ Using Eq. (5), we plot in Fig. 1 the change in the vibrational contribution $\Delta F^{\mathrm{vib}}$ to the surface free energy [dashed light (blue) and black lines]. The vibrational contribution of the bulk to the total energy is shown as well [solid midgray (red) line]. The figure shows that the change in the vibrational energy less than $4 \mathrm{meV} / \AA^{2}$ for temperatures in a range between 0 and $1000 \mathrm{~K}$. These values of $\Delta F^{\mathrm{vib}}$ are comparable to those reported for $\mathrm{RuO}_{2}$ (Ref. 48) and $\mathrm{Cu}_{2} \mathrm{O}{ }^{55}$ In order to cross-check this result, we also estimated the vibrational contribution to the surface free energy by using the calculated frequency spectrum (at the $\Gamma$-point): $\quad \Delta F^{\mathrm{vib}}=F_{\text {Surf }}^{\mathrm{vib}}-F_{\mathrm{Bulk}}^{\mathrm{vib}}$. Here $F^{\mathrm{vib}}\left(T, \omega_{i}\right)=1 / 2 \hbar \omega_{i}$ $+k_{B} T \ln \left(1-e^{-\hbar \omega_{i} / k_{B} T}\right)$ is the vibrational free energy for a given mode at frequency $\omega_{i}$. The result is shown in Fig. 2 where it can be seen that $\Delta F^{\mathrm{vib}}$ is less than $5 \mathrm{meV}$ for the temperature range up to $1000 \mathrm{~K}$. Thus, for this system, the vibrational contributions are negligible and are neglected in the present work.

\section{B. Bulk cerium and the oxygen molecule}

We first calculate the properties of bulk Ce and the $\mathrm{O}_{2}$ molecule as they are required to calculate the bulk properties of $\mathrm{CeO}_{2}$ and $\mathrm{Ce}_{2} \mathrm{O}_{3}$, such as the heat of formation and cohesive energy.
Bulk cerium metal forms in three crystalline structures: The $\alpha, \beta$, and the $\gamma$-phases. For the temperatures and pressures we consider in the present study, the $\alpha$-phase is the stable one. ${ }^{56}$ The calculated optimized lattice constants of $\alpha$-Ce (face-centered cubic structure) are $4.74 \AA$ (GGA) and $4.53 \AA$ (LDA), while from PAW-GGA and -LDA calculations, the values are 4.73 and $4.51 \AA$, respectively. ${ }^{22}$ An earlier DFT-GGA and -LDA study obtained 4.71 and $4.49 \AA$, respectively. ${ }^{57}$ The experimental value is $4.83 \AA \AA^{58}$ We also calculate the bulk modulus to be $57.6 \mathrm{GPa}$ (LDA) and 37.1 GPa (GGA). Reported values in the literature are $60.5 \mathrm{GPa}(\mathrm{LDA})$ and $48.7 \mathrm{GPa}(\mathrm{GGA}),{ }^{57}$ and the experimental value is $29.0 \mathrm{GPa}^{59}$ As can be seen, both GGA and LDA give values of the lattice parameters smaller than the experimental value. This could, in part, be due to the inadequacies of DFT to describe the electron-correlation-driven volume changes in Ce. ${ }^{60-62}$ The mechanism behind this complicated "volume collapse" is still very much a subject of debate. ${ }^{60,61}$ A detailed discussion of this anomalous phase change is beyond the scope of this work and thus, we refer readers to Ref. 61 and references therein for more details. Likewise, the poor description of the bulk modulus is a direct consequence of the above-mentioned phenomenon. Nevertheless, our calculated DFT (for both LDA and GGA) results are in very good agreement with other reported theoretical values as cited above.

Spin unrestricted DFT-GGA calculations using nonspherical densities are performed to study the oxygen atom and molecule. The real-space cutoff for the calculation of both the oxygen atom and molecule is 20 bohrs, using the largest basis set available in the $\mathrm{DMol}^{3}$ code. The binding energy of $\mathrm{O}_{2}$ is calculated to be $3.04 \mathrm{eV} / \mathrm{O}$ atom, while the bond length and the vibrational frequency are $1.22 \AA$ and 
TABLE II. Bulk properties of $\mathrm{Ce}_{2} \mathrm{O}_{3}$ calculated using the LDA and GGA-PBE. $a(\AA), B_{0}(\mathrm{GPa}), E_{\text {coh }}(\mathrm{eV})$, and $\Delta H_{f}(\mathrm{eV})$ refers to the bulk lattice constant (with the $c / a$ value given in brackets), bulk modulus, cohesive energy, and heat of formation, respectively. The indirect $\mathrm{O}-2 p-\mathrm{Ce}-4 f$ bandgap is represented by $E_{g}$ in $\mathrm{eV}$. Results of other first-principles calculations and experiment are listed for comparison. The full-potential augmented plane wave approach is denoted as FPLAPW. The Gaussian local-orbital method is abbreviated by GTO while PAW refers to the PAW method. The hybrid DFT functionals due to Heyd-Scuseria-Ernzerhof is referred to as DFT-HSE, ${ }^{66}$ and Adamo and Barone as PBE0,${ }^{67}$ while the meta-GGA due to Tao et al. is referred to as TPSS. (68) NM, FM, and AFM indicate the nonmagnetic, ferromagnetic, and antiferromagnetic cases, respectively.

\begin{tabular}{|c|c|c|c|c|c|}
\hline & $a(c / a)$ & $B_{0}$ & $E_{\mathrm{coh}}$ & $\Delta H_{f}$ & $E_{g}$ \\
\hline $\mathrm{DMol}^{3}(\mathrm{LDA})[\mathrm{FM}]^{\mathrm{a}}$ & $3.76(1.57)$ & 146.7 & -42.61 & -17.26 & 0.0 \\
\hline $\mathrm{DMol}^{3}(\mathrm{LDA})[\mathrm{AFM}]^{\mathrm{a}}$ & $3.73(1.60)$ & 154.9 & -42.56 & -17.21 & 0.0 \\
\hline $\mathrm{DMol}^{3}(\mathrm{PBE})[\mathrm{FM}]^{\mathrm{a}}$ & $3.85(1.58)$ & 123.8 & -36.60 & -15.95 & 0.0 \\
\hline $\mathrm{DMol}^{3}(\mathrm{PBE})[\mathrm{AFM}]^{\mathrm{a}}$ & $3.86(1.58)$ & 113.9 & -36.50 & -15.85 & 0.0 \\
\hline PAW-LDA[FM $]^{\mathrm{b}}$ & $3.77(1.56)$ & 138.0 & -40.41 & $\ldots$ & $\cdots$ \\
\hline PAW-LDA[AFM] $]^{\mathrm{b}}$ & $3.73(1.59)$ & 138.0 & -40.35 & -18.07 & 0.0 \\
\hline PAW-PBE[FM] $]^{b}$ & $3.83(1.59)$ & 114.0 & -34.80 & $\cdots$ & $\cdots$ \\
\hline PAW-PBE[AFM $]^{\mathrm{b}}$ & $3.73(1.59)$ & 138.0 & -34.72 & -16.30 & 0.01 \\
\hline FPLAPW-PBE[FM $]^{\mathrm{b}}$ & $3.85(1.57)$ & 113.0 & -34.60 & -16.47 & 0.0 \\
\hline $\mathrm{PAW}-\mathrm{LDA}+U[\mathrm{FM}]^{\mathrm{b}}$ & $3.87(1.53)$ & $\cdots$ & $\cdots$ & $\cdots$ & $\cdots$ \\
\hline PAW-LDA $+U[\mathrm{AFM}]^{\mathrm{b}}$ & $3.86(1.54)$ & $\cdots$ & $\cdots$ & $\cdots$ & 2.4 \\
\hline $\mathrm{PAW}-\mathrm{PBE}+U[\mathrm{FM}]^{\mathrm{b}}$ & $3.92(1.58)$ & $\cdots$ & $\cdots$ & $\cdots$ & $\cdots$ \\
\hline $\mathrm{PAW}-\mathrm{PBE}+U[\mathrm{AFM}]^{\mathrm{b}}$ & $3.92(1.58)$ & $\cdots$ & $\cdots$ & $\cdots$ & 2.6 \\
\hline PAW-PBE0 $[\mathrm{FM}]^{\mathrm{b}}$ & $3.87(1.57)$ & $\cdots$ & -32.44 & $\cdots$ & $\cdots$ \\
\hline PAW-PBE0[AFM $]^{\mathrm{b}}$ & $3.87(1.57)$ & $\cdots$ & -32.45 & -19.18 & 3.5 \\
\hline PAW-HSE[FM] $]^{\mathrm{b}}$ & $3.87(1.57)$ & $\cdots$ & -33.54 & $\cdots$ & $\cdots$ \\
\hline PAW-HSE $[\mathrm{AFM}]^{\mathrm{b}}$ & $3.87(1.57)$ & $\cdots$ & -33.55 & -18.85 & 2.5 \\
\hline GTO-LDA[FM $]^{c}$ & $3.76(1.56)$ & $\cdots$ & $\cdots$ & $\cdots$ & 0.0 \\
\hline GTO-LDA[AFM $]^{c}$ & $3.78(1.55)$ & $\cdots$ & $\cdots$ & $\ldots$ & 0.0 \\
\hline GTO-PBE[FM $]^{c}$ & $3.88(1.54)$ & $\cdots$ & $\cdots$ & $\cdots$ & 0.0 \\
\hline GTO-PBE[AFM $]^{c}$ & $3.88(1.57)$ & $\cdots$ & $\cdots$ & $\cdots$ & 0.3 \\
\hline GTO-TPSS $[\mathrm{FM}]^{\mathrm{c}}$ & $3.88(1.55)$ & $\cdots$ & $\cdots$ & $\cdots$ & $\cdots$ \\
\hline GTO-TPSS[AFM $]^{c}$ & $3.88(1.55)$ & $\cdots$ & $\cdots$ & $\cdots$ & 0.5 \\
\hline GTO-HSE[AFM $]^{c}$ & $3.86(1.55)$ & $\cdots$ & $\cdots$ & $\cdots$ & 3.2 \\
\hline
\end{tabular}

${ }^{\mathrm{a}}$ This work.

${ }^{\mathrm{b}}$ Reference 22.

${ }^{\mathrm{c}}$ Reference 20 .

$1580 \mathrm{~cm}^{-1}$, respectively. These values are in good agreement with other theoretical results. ${ }^{45,63,64}$ The corresponding experimental values are $2.56 \mathrm{eV} / \mathrm{O}$ atom, $1.21 \AA$, and $1580 \mathrm{~cm}^{-1} .^{65}$ The typical overestimation of DFT-GGA is observed in the binding energy. The calculated values presented here are indicative of well-converged DFT-GGA calculations, and since our interest lies mainly in the relative stability of various structures, this overbinding will not affect the qualitative conclusions in this paper.

\section{Bulk properties of $\mathrm{CeO}_{2}$ and $\mathrm{Ce}_{2} \mathrm{O}_{3}$}

Cerium oxide forms in a fluorite structure $\left(\mathrm{CeO}_{2}\right)$, in a hexagonal structure $\left(\mathrm{Ce}_{2} \mathrm{O}_{3}\right)$ and in a cubic structure $\left(\mathrm{Ce}_{2} \mathrm{O}_{3}\right)$ with space groups $F m \overline{3} m,{ }^{35} P \overline{3} m 1,{ }^{35}$ and $I a \overline{3},{ }^{44}$ respectively. In this work, we investigate the fluorite and the hexagonal structure which are depicted in Figs. 3(a) and 3(b), respectively. For $\mathrm{CeO}_{2}$, the $\mathrm{Ce}$ atom is coordinated to eight oxygen atoms, while in the $\mathrm{Ce}_{2} \mathrm{O}_{3}$ structure, each $\mathrm{Ce}$ is coordinated to seven oxygen atoms. Our DFT calculations find $\mathrm{CeO}_{2}$ is a semiconductor with indirect $\mathrm{O}-2 p-\mathrm{Ce}-4 f$ band gaps of $1.97 \mathrm{eV}$ (GGA) and $2.11 \mathrm{eV}$ (LDA). Using PAWs as a basis set, Jiang et $a l .{ }^{38}$ found rather similar underestimated values of $1.72 \mathrm{eV}(\mathrm{GGA})$ and $1.89 \mathrm{eV}$ (LDA) for the O-2p-Ce- $4 f$ band gap. Upon applying an effective Hubbard potential of $U=6.3 \mathrm{eV}$ for both LDA and GGA calculations, not surprisingly, the band gap was "improved" to $2.54 \mathrm{eV}$, bringing it much closer to the experimental value of $3.00 \mathrm{eV} .^{24}$ Similarly, using a Gaussian local orbital basis set and a small-core relativistic Ce pseudopotential, Hay et al. ${ }^{20}$ reported a LDA band gap of $1.6 \mathrm{eV}$ and the corresponding GGA value of $1.7 \mathrm{eV}$. The authors further tested the next "rank" of functionals-the meta-GGA, namely, the "Tao- PerdewStaroverov-Scuseria" (TPSS) functional, ${ }^{68}$ only to find that the band gap was increased by a mere $0.1 \mathrm{eV}$ as compared to their GGA value. However, using the HSE hybrid DFT functional, the authors obtained a much better agreement with experiment, overestimating the experimental band $\operatorname{gap}^{24}$ $(3.0 \mathrm{eV})$ slightly by $0.3 \mathrm{eV} .^{20}$ This was further confirmed by the work of Da Silva et $a l .{ }^{22}$ where the PAW formalism was employed with the different ranks of functionals, namely, the $\mathrm{LDA}$ and $\mathrm{GGA}(\mathrm{PBE}), \mathrm{DFT}+U$, and hybrid DFT functionals of HSE and PBE0. ${ }^{67}$ Our calculated indirect $\mathrm{O}-2 p-\mathrm{Ce}-4 f$ band gap is very much in accord with other reported DFT values, with the LDA value consistently larger than the corresponding GGA value. Our slightly larger values could be due to an all-electron description (i.e., without the use of 

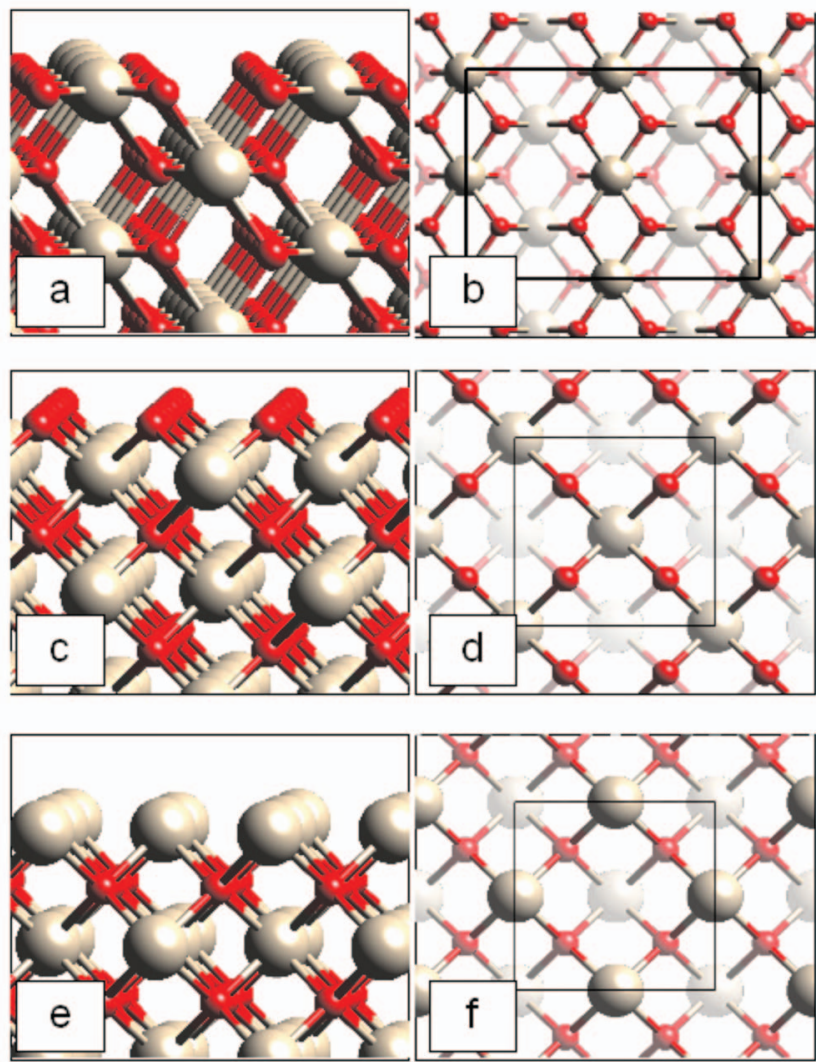

FIG. 4. Side (left) and top (right) views of various terminations of $\mathrm{CeO}_{2}(110)$ and $\mathrm{CeO}_{2}(100)$ surfaces: (a) and (b) are for the stoichiometric (110) surface, (c) and (d) are $\mathrm{CeO}_{2}(100): \mathrm{O}$, and (e) and (f) are $\mathrm{CeO}_{2}(100)$ : $\mathrm{Ce}$. The surface unit cells used in the calculations are indicated: $(2 \times 2)$ for $\mathrm{CeO}_{2}(110)$ and $(\sqrt{2} \times \sqrt{2}) R 45^{\circ}$ for $\mathrm{CeO}_{2}(100): \mathrm{O}$ and $\mathrm{CeO}_{2}(100): \mathrm{Ce}$.

pseudopotentials or the frozen core approximation). A summary of the bulk properties of $\mathrm{CeO}_{2}$ is listed in Table $\mathrm{I}$.

Experimentally, the sesquioxide of cerium, $\mathrm{Ce}_{2} \mathrm{O}_{3}$, is known to be a semiconductor with a band gap of $2.40 \mathrm{eV}$. ${ }^{69}$ However, it is well established that unless proper cancellation of the Coulomb self-interaction in the density functionals is taken into account, conventional DFT calculations will fail to capture the insulating nature of these so-called MottHubbard insulators, resulting a metallic character (for $\mathrm{Ce}_{2} \mathrm{O}_{3}$ ). As a consequence, the magnetic behavior is also wrongly described. To overcome this, it is usual to employ some form of approaches that goes beyond standard DFT-GGA. ${ }^{11-13,22,39}$ Using the hybrid DFT approach, e.g., outlined in Refs. 20 and 22, it is shown to improve the description of such class of materials, however, the associated computational cost is still rather daunting. In this work we report the properties of bulk $\mathrm{Ce}_{2} \mathrm{O}_{3}$ within the conventional DFT framework (see Table II for more details). We have not corrected for the self-interaction error in our work as our primary objective is to focus on the relative thermodynamic stability of $\mathrm{CeO}_{2}$ surfaces, argued on the grounds of energetics (at least for a qualitative trend). Furthermore, current methods, such as the DFT $+U$ method, are somewhat controversial. Hence, we have chosen a more transparent (yet tractable) approach of conventional DFT to offer a first understanding of ceria surfaces.
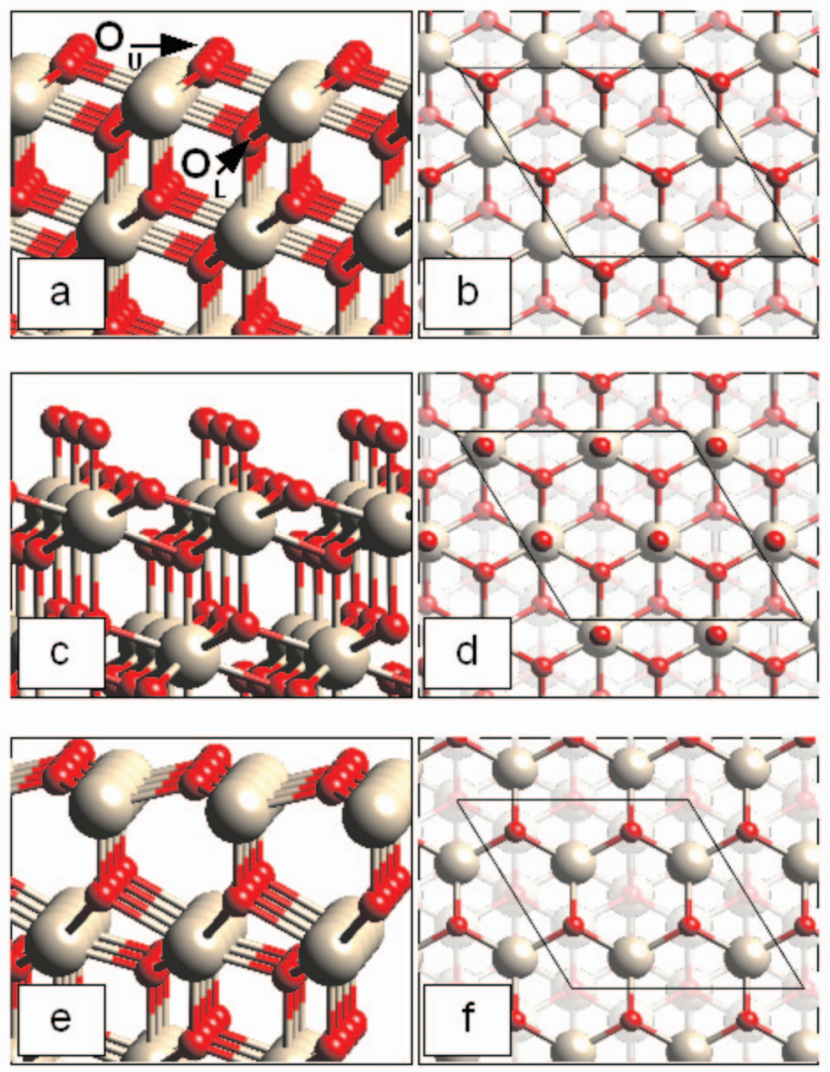

FIG. 5. Side (left) and top (right) views of various terminations of the $\mathrm{CeO}_{2}$ (111) surfaces: (a) and (b) are the stoichiometric $\mathrm{CeO}_{2}(111)$ surface, (c) and (d) are $\mathrm{CeO}_{2}(111): \mathrm{O}$, and (e) and (f) are $\mathrm{CeO}_{2}(111): \mathrm{Ce}$ (relaxed surface structure). The surface $(2 \times 2)$ unit cells used in the calculations are indicated.

Our results for bulk $\mathrm{Ce}_{2} \mathrm{O}_{3}$ are consistent with other reported DFT values: We find that it is a metal, where the lattice constants $a$ are $3.86 \AA(c / a=1.56)$ and $3.77 \AA$ $(c / a=1.56)$ for the GGA and LDA, respectively. Da Silva et $a .^{22}$ reported the corresponding values of $3.83 \AA$ $(c / a=1.59)$ and $3.77 \AA(c / a=1.56)$ for the GGA and LDA, respectively. The authors of Ref. 22 found that using hybrid DFT and LDA $+U$ gives comparable lattice constants to the DFT-derived values, while GGA $+U$ worsens the lattice parameters, giving too large $a$ and $c$ values. Our calculated DFT-GGA (LDA) heat of formation per formula unit $[-15.95 \mathrm{eV}(-17.26)]$ is found to be smaller than both re-

TABLE III. First interlayer distance $d_{12}$ and corresponding relative interlayer relaxation (compared to the bulk values) of the various $\mathrm{CeO}_{2}$ surfaces considered. Positive values indicate a contraction of the distance.

\begin{tabular}{lcc}
\hline \hline & $d_{12}(\AA)$ & $\begin{array}{c}\text { Relative interlayer } \\
\text { relaxation } \\
(\%)\end{array}$ \\
\hline $\mathrm{CeO}_{2}(111)$ & 1.540 & 0.6 \\
$\mathrm{CeO}_{2}(111): \mathrm{Ce}$ & 2.149 & 5.0 \\
$\mathrm{CeO}_{2}(111): \mathrm{O}$ & 1.380 & 3.0 \\
$\mathrm{CeO}_{2}(110)$ & 1.760 & 0.7 \\
$\mathrm{CeO}_{2}(100): \mathrm{O}$ & 1.201 & 1.0 \\
$\mathrm{CeO}_{2}(100): \mathrm{Ce}$ & 1.091 & 1.3 \\
$\mathrm{Ce}_{2} \mathrm{O}_{3}(0001): \mathrm{O}$ & 0.740 & 1.0 \\
\hline \hline
\end{tabular}



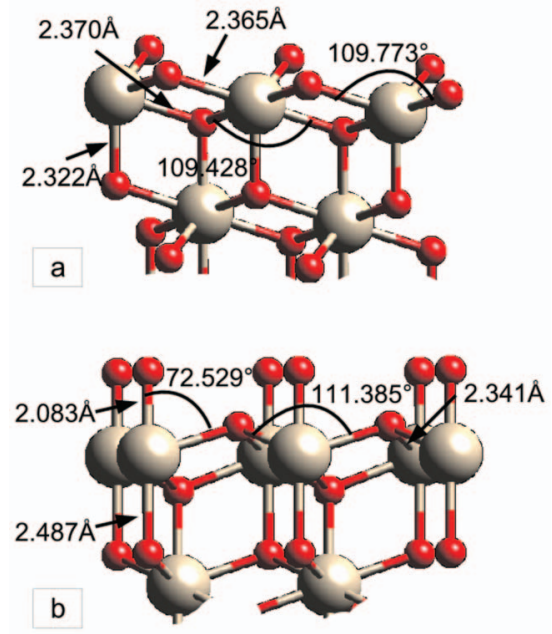

FIG. 6. Local atomic structure of the various $\mathrm{CeO}_{2}(111)$ surfaces: (a) $\mathrm{CeO}_{2}(111)$; (b) $\mathrm{CeO}_{2}(111)$ : O. Various bond lengths and angles are indicated.

ported experimental $^{70}$ and DFT (Ref. 22) values of -18.62 and $-16.30 \mathrm{eV}(-18.07)$, respectively. However, comparing our calculated heat of formation per $\mathrm{O}$ atom (i.e., dividing the values given above by a factor of 3) with that of Ref. 22, the percentage difference is only marginal, i.e., about $2 \%-$ $4 \%$, and will not have a significant effect on the qualitative trends discussed.

\section{D. $\mathrm{CeO}_{2}$ low-index ideal bulk surface terminations}

We now consider the properties of the low-index surfaces, (100), (110), and (111) of $\mathrm{CeO}_{2}$, each with a number of different terminations. For $\mathrm{CeO}_{2}(100)$, there is an O-terminated surface, denoted $\mathrm{CeO}_{2}(100): \mathrm{O}$ [shown in Figs. 4(c) and 4(d)], and a Ce-terminated one, $\mathrm{CeO}_{2}(100): \mathrm{Ce}$, (shown in Figs. 4(e) and 4(f)). For $\mathrm{Ce}_{2} \mathrm{O}(110)$ we consider the stoichiometric termination shown in Figs. 4(a) and 4(b). Similarly to $\mathrm{CeO}_{2}(100)$, the $\mathrm{CeO}_{2}(111)$ surface can either be O-terminated, $\mathrm{CeO}_{2}(111): \mathrm{O}$ [Figs. 5(c) and 5(d)], or Ceterminated, $\mathrm{CeO}_{2}(111)$ : $\mathrm{Ce}$ [Figs. 5(e) and 5(f)]. The third termination [hereafter labeled as $\mathrm{CeO}_{2}(111)$, Figs. 5(a) and $5(\mathrm{~b})$ ] can be understood as cutting the stacking of $\mathrm{O}-\mathrm{Ce}-\mathrm{O}$ trilayers along the [111] direction between consecutive trilayers, thus resulting in a stoichiometric (111) surface. Of the six different surface terminations considered, two of them are stoichiometric, while the others have either an excess of $\mathrm{Ce}$ or $\mathrm{O}$ atoms at the surface, and thus belong to the class of "polar" surfaces. 71

Table III shows the first interlayer distances for the various surfaces and the corresponding percentage relaxation with respect to the interlayer spacing of the bulk, i.e., it is calculated as $d=\left(d_{i j}-d_{0}\right) / d_{i j} \times 100 \%$, where $d_{i j}$ is the interlayer spacing between the $i$ th and $j$ th atomic layers [for the (110) and the (100) surfaces] and $d_{0}$ is the bulk interlayer spacing. For the (111) surfaces, $d_{i j}$ is defined as the distance between the last layer of the first trilayer and the first layer of the second trilayer. Interlayer relaxation between the second and third layers is found to be negligible $\left(d_{23} \leqslant 1 \%\right)$.

The local atomic structures are also depicted in Figs. 6 and 7 for the $\mathrm{CeO}_{2}(111)$ surfaces, and in Fig. 8 for the
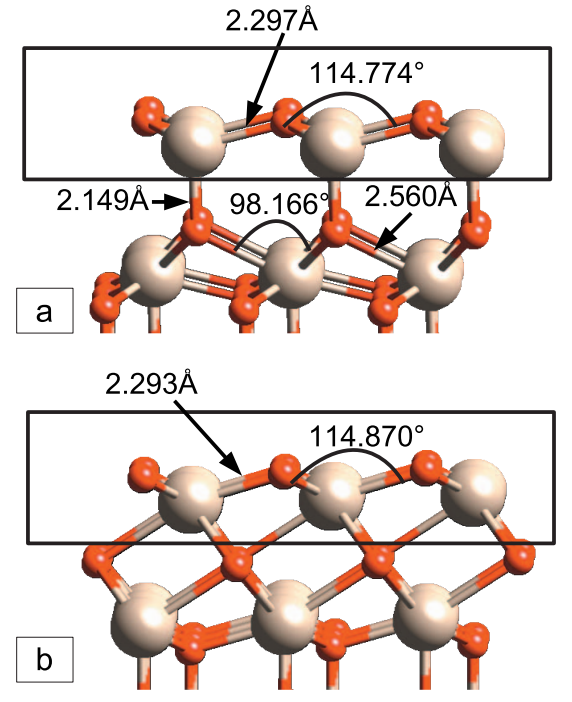

FIG. 7. Local atomic structure of the various $\mathrm{CeO}_{2}(111)$ surfaces: (a) $\mathrm{CeO}_{2}(111)$ : $\mathrm{Ce}$ and (b) $\mathrm{Ce}_{2} \mathrm{O}_{3}(0001)$. Various bond lengths and angles are indicated. The rectangles highlight the similar structure of the $\mathrm{Ce}_{2} \mathrm{O}_{3}(0001)$ and $\mathrm{CeO}_{2}(111)$ : Ce surface terminations.

$\mathrm{CeO}_{2}(110)$ and $\mathrm{CeO}_{2}(100)$ surfaces, where values of the bond distances $d_{\mathrm{Ce}-\mathrm{O}}$ for atoms in the outermost layers and the angles $\angle \mathrm{O}-\mathrm{Ce}-\mathrm{O}$ are given. For the $\mathrm{CeO}_{2}(111)$ : O surface termination, there is a contraction of about $3 \%$ of the first interlayer spacing as compared to the bulk. The $\mathrm{Ce}-\mathrm{O}$ bond lengths involving the surface $\mathrm{O}$ atoms are $11 \%$ shorter than the bulk distances due to a reduced coordination [Fig. 6(b)]. For the $\mathrm{CeO}_{2}(111)$ : Ce termination, there is a significant surface reconstruction where we find that the optimized geometry actually becomes an O-terminated surface. In particular, there is a huge outward displacement of the oxygen atoms in the second layer of $\sim 1.2 \AA$ so that they, instead of the $\mathrm{Ce}$

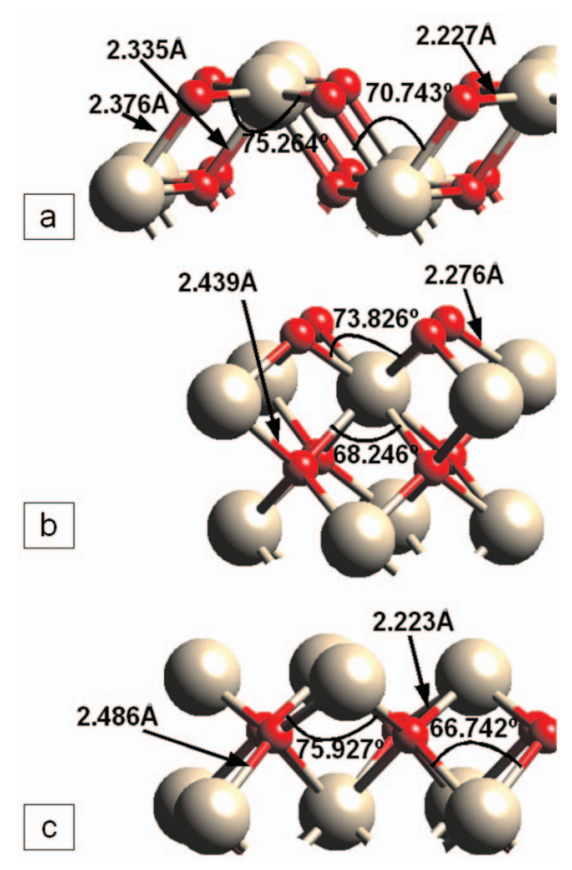

FIG. 8. Local atomic structure of the $\mathrm{CeO}_{2}(110)$ and $\mathrm{CeO}_{2}(100)$ surfaces: (a) $\mathrm{CeO}_{2}(110)$, (b) $\mathrm{CeO}_{2}(100): \mathrm{O}$, and (c) $\mathrm{CeO}_{2}(100): \mathrm{Ce}$. Various bond lengths and angles are indicated. 
atoms, become the uppermost surface atoms [see Fig. 7(a)]. Interestingly, at a first glance, the cross section of the first two atomic layers of the $\mathrm{CeO}_{2}(111)$ : Ce surface resembles that of the $\mathrm{Ce}_{2} \mathrm{O}_{3}(0001)$ surface [compare Figs. 7(a) and 7(b)]. The calculated bond angles and distances of the first two atomic layers of these two surfaces are indeed found to be very similar. Restricting to just the first two atomic layers, the $d_{\mathrm{Ce}-\mathrm{O}}$ of $\mathrm{CeO}_{2}(111): \mathrm{Ce}$ and $\mathrm{Ce}_{2} \mathrm{O}_{3}(0001)$ are found to be 2.297 and $2.283 \AA$, respectively, while the corresponding angles $\angle \mathrm{O}-\mathrm{Ce}-\mathrm{O}$ for both these surfaces are $114.8^{\circ}$. Furthermore, if one is to overlay the surface unit cell of $\mathrm{Ce}_{2} \mathrm{O}_{3}(0001)$ over that of $\mathrm{CeO}_{2}(111)$ : $\mathrm{Ce}$, both surface areas overlap almost commensurately, with the surface lattice vectors-deviating by barely less than $5 \times 10^{-4} \AA$. This is indicative that the $\mathrm{CeO}_{2}(111)$ : $\mathrm{Ce}$ structure could be a precursor to the initial stages of a phase transformation to $\mathrm{Ce}_{2} \mathrm{O}_{3}(0001)$. Below we will investigate the relative stability of this structure and the gaseous environmental conditions under which it may be realized.

The $\mathrm{CeO}_{2}(110)$ surface exhibits only a small contraction compared to the bulk value $(\sim 0.7 \%)$ of the first interlayer spacing. The $\mathrm{Ce}-\mathrm{O}$ bond distance at the surface is $d_{\mathrm{Ce}-\mathrm{O}}=2.23 \AA$, slightly reduced compared to the bulk value (of $2.37 \AA$ ) [see Fig. 8(a)]. Also there is a small contraction of the bond length between the Ce atom at the surface and of the $\mathrm{O}$ atom in the second layer $(2.33 \AA)$ and of the $\mathrm{O}$ atom at the surface and the Ce atom in the second layer $(2.38 \AA)$. For the $\mathrm{CeO}_{2}(100)$ surfaces, Figs. 8(b) and 8(c), the upper $\mathrm{Ce}-\mathrm{O}$ bond lengths are contracted relative to the bulk values [2.28 and $2.22 \AA$ for $\mathrm{CeO}_{2}(100): \mathrm{O}$ and $\left.\mathrm{CeO}_{2}(100): \mathrm{Ce}\right]$, namely by $-3.9 \%$ and $-6.1 \%$, respectively.

\section{E. $\mathrm{CeO}_{2}$ low-index terminations with surface defects}

In addition to the (initially) ideally bulk terminated surfaces, we consider various structures containing defects, created by removing from (or adding to) the ideal terminated structures, single oxygen, or cerium atoms.

For the $\mathrm{CeO}_{2}(110)$ surface, we consider $\mathrm{Ce}$ and $\mathrm{O}$ vacancies. When a Ce vacancy $V_{\mathrm{Ce}}$ is introduced in the first atomic layer [see Figs. 9(a) and 9(b)], there is the formation of an $\mathrm{O}-\mathrm{O}$ bond at the surface with a bond distance of $d_{\mathrm{O}-\mathrm{O}}=1.44 \AA$ and an associated displacement of the two $\mathrm{O}$ atoms of about $0.40 \AA$ with respect to their positions without the vacancy (Fig. 10).

For the $\mathrm{CeO}_{2}(100): \mathrm{O}+V_{\mathrm{Ce}}$ surface there is a huge displacement of the uppermost oxygen atoms where they form a square planar cluster over surface $\mathrm{Ce}$ atoms [see Figs. 11(a) and 11(b)]. The nearest-neighbor $\mathrm{O}-\mathrm{O}$ distance in the cluster is $1.53 \AA$ and the distance of the $\mathrm{O}$ atoms to the $\mathrm{Ce}$ atoms below is $3.09 \AA$ [see Fig. 11(c)], which is $\sim 25 \%$ larger than the bulk value.

Oxygen clustering occurs for the $\mathrm{CeO}_{2}(111): \mathrm{O}$ system when a $\mathrm{Ce}$ vacancy is created [i.e., $\mathrm{CeO}_{2}(111): \mathrm{O}+V_{\mathrm{Ce}}$ ]. In particular, a clustering of six oxygen atoms is observed with an $\mathrm{O}-\mathrm{O}$ bond length of $d_{\mathrm{O}-\mathrm{O}}=1.48 \AA$, which is arranged in an "arm chair" configuration [see Figs. 12(a)-12(c)]. For the $\mathrm{CeO}_{2}(111)$ surface with an $\mathrm{O}$ vacancy [see Figs. 13(c) and $13(\mathrm{~d})]$ there is a displacement of the oxygen atoms in the
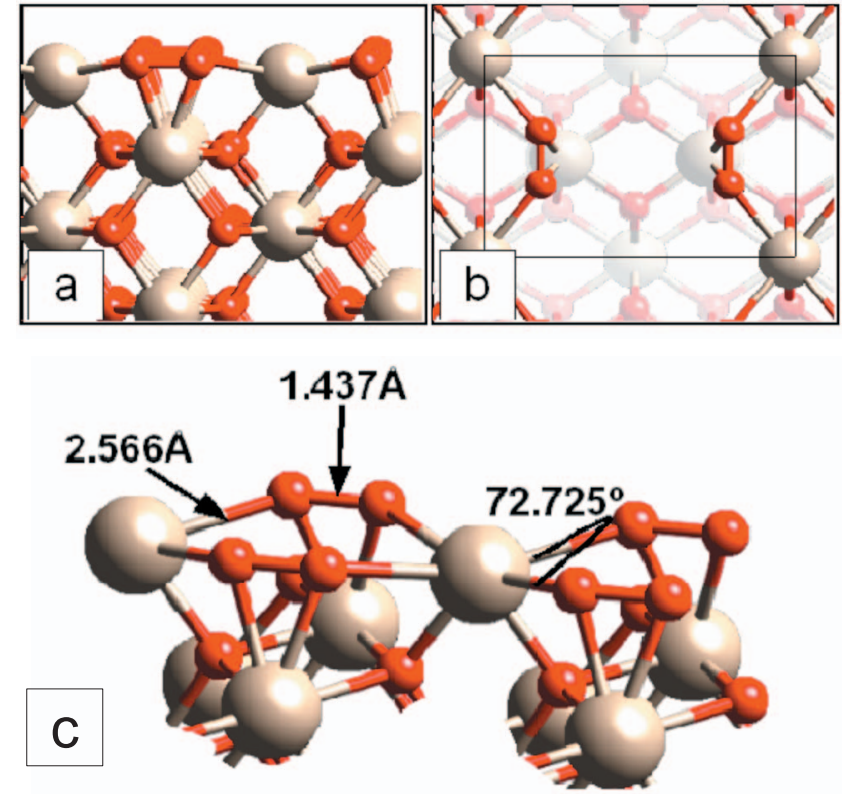

FIG. 9. Side view (a), top view (b), and local atomic geometry (c) of $\mathrm{CeO}_{2}(110)+V_{\mathrm{Ce}}$. The horizontal and vertical lines of the unit cell indicated in (b) correspond to the [110] and [001] directions, respectively. The side view in (a) is for the $(100)$ plane. The $(2 \times 1)$ surface unit cells is indicated (b). Various bond lengths and angles are indicated (c).

second layer toward the oxygen vacancy of $\sim 0.4 \AA$ and a displacement of the cerium atom away from the vacancy of $\sim 0.2 \AA$ [see Fig. 13(f)]. For the $\mathrm{CeO}_{2}(111)+V_{\mathrm{Ce}}$ surface [Figs. 13(a) and 13(b)], the $\mathrm{O}$ atoms neighboring the $\mathrm{Ce}$ vacancy are displaced toward it by $\sim 0.4 \AA$ [see Figs. 13(e)].

For the stoichiometric $\mathrm{CeO}_{2}(111)$ surface we also considered a subsurface $\mathrm{O}$ atom in an interstitial site [Fig. 14(a)]. Upon relaxing the structure, there is an outward displacement of the outermost surface oxygen atom $\left(\mathrm{O}_{t}\right)$ directly above the interstitial oxygen atom $\left(\mathrm{O}_{i}\right)$, resulting in the formation of a bond between these two oxygen atoms with a bond length of $1.44 \AA$ (compared to $1.22 \AA$ for the $\mathrm{O}_{2}$ mol-

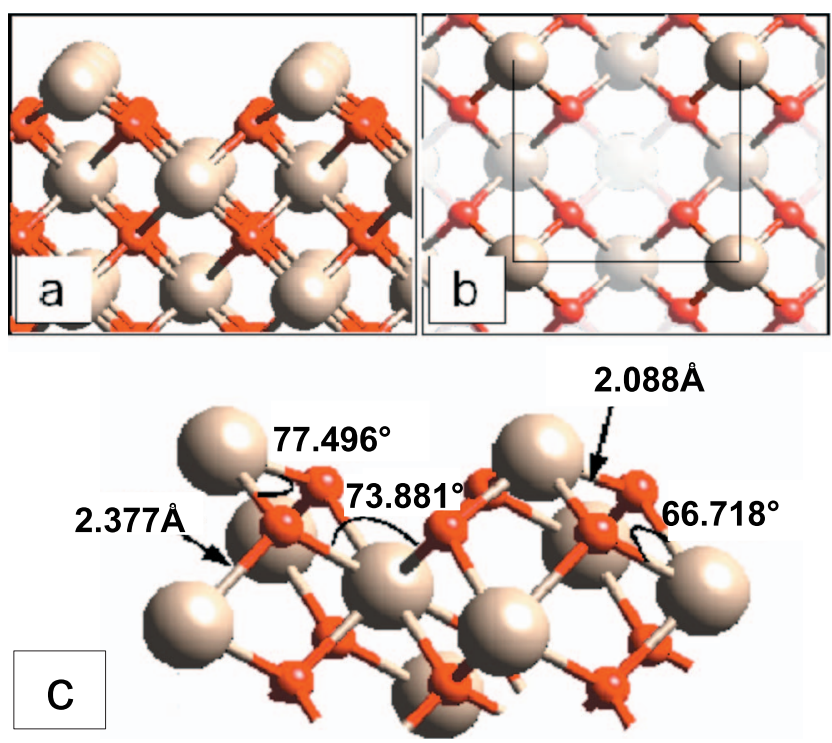

FIG. 10. Side view (a), top view (b), and local atomic geometry (c) of the $\mathrm{CeO}_{2}(100): \mathrm{Ce}+V_{\mathrm{Ce}}$. The $(\sqrt{2} \times \sqrt{2}) R 45^{\circ}$ surface unit cells used in the calculations are shown (b). Various bond lengths and angles are indicated (c). 

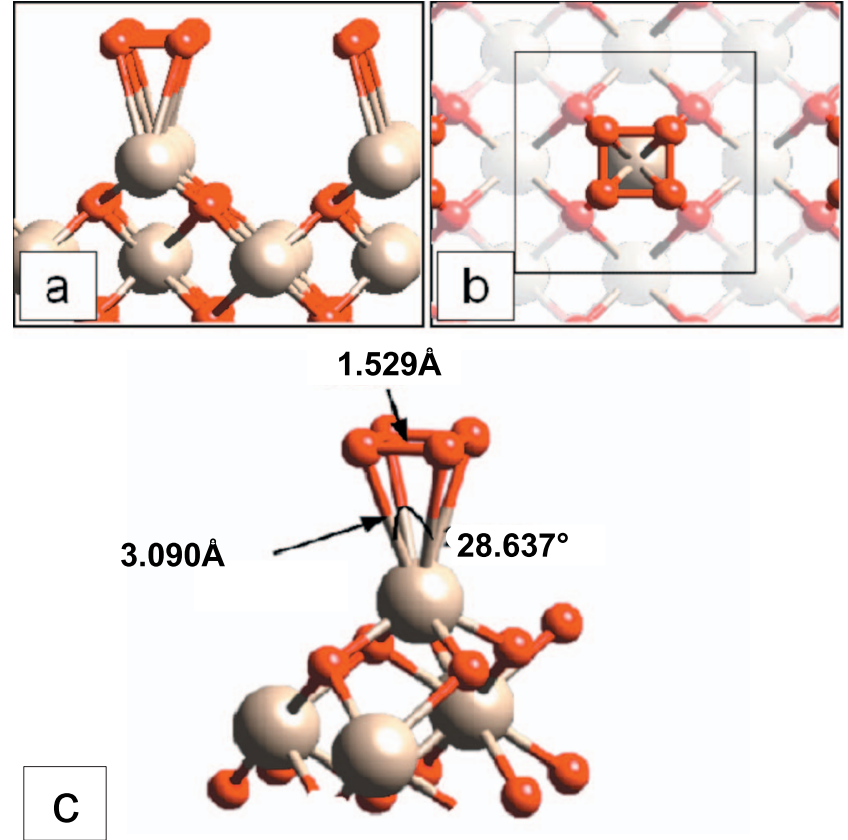

FIG. 11. Side view (a), top view (b), and local atomic geometry (c) of the $\mathrm{CeO}_{2}(100): \mathrm{O}+V_{\mathrm{Ce}}$ surface. The $(\sqrt{2} \times \sqrt{2}) R 45^{\circ}$ surface unit cells used in the calculations are shown (b). Various bond lengths and angles are indicated (c).

ecule), and a consequent increase in the $\mathrm{Ce}-\mathrm{O}$ bond length, between $\mathrm{O}_{t}$ and the cerium atom at the surface, of $\sim 10 \%$ compared to the bulk value [see Fig. 14(c)]. On increasing the number of interstitial oxygen atoms (by adding a second $\mathrm{O}$ atom in the surface unit cell), the resulting geometry is similar to the one previously described; bond distances and angles change by less than $1 \%$ (Table IV). The surface energy with this higher concentration of interstitial $\mathrm{O}$ atoms is less favorable (by $0.02 \mathrm{eV} / \AA^{2}$ ), indicating a repulsive interaction between these interstitial $\mathrm{O}$ atoms.
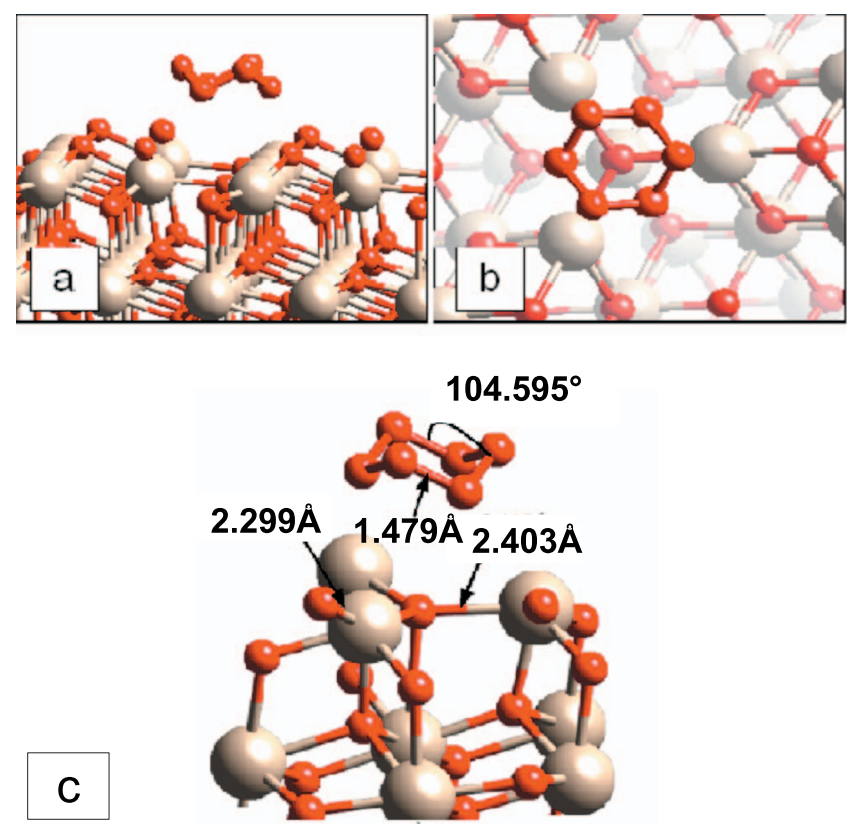

FIG. 12. Side view (a), top view (b), and local atomic geometry (c) of the $\mathrm{CeO}_{2}(111): \mathrm{O}+V_{\mathrm{Ce}}$ surface. Various bond lengths and angles are indicated (c).
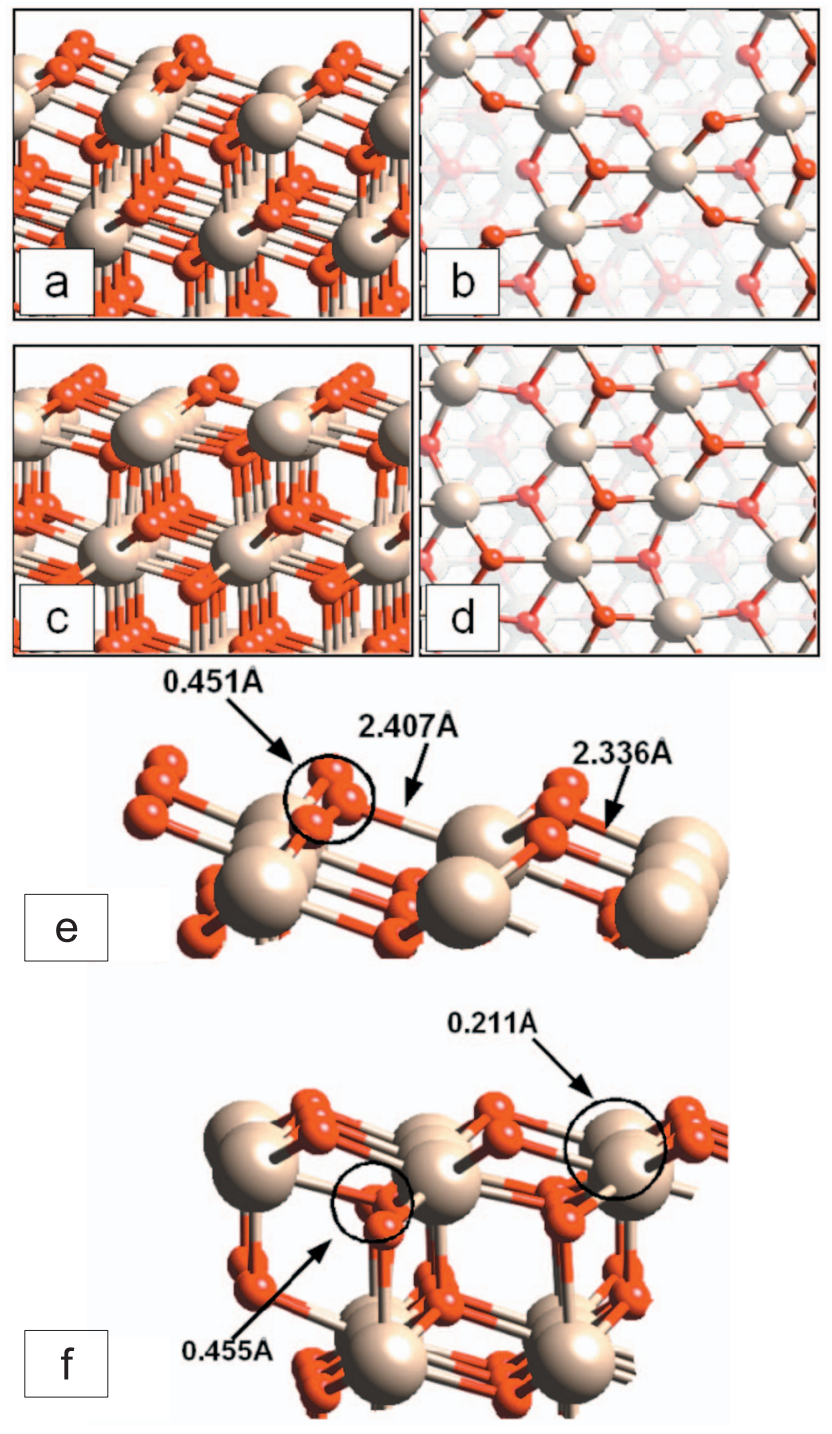

FIG. 13. Side view, top view, and local atomic geometry of the $\mathrm{CeO}_{2}(111)$ surfaces with defects: (a) and (b) $\mathrm{CeO}_{2}(111)+V_{\mathrm{Ce}}$ and (c) and (d) $\mathrm{CeO}_{2}(111)+V_{\mathrm{O}}$. (e) and (f) represent the local atomic geometry of $\mathrm{CeO}_{2}(111)+V_{\mathrm{Ce}}$ and $\mathrm{CeO}_{2}(111)+V_{\mathrm{O}}$, respectively.

\section{F. Energetics and thermodynamics}

We now proceed to analyze the stability of these different oxide surfaces when in contact with an oxygen environment, characterized by a given $\mathrm{O}$ chemical potential. We first plot the surface Gibbs free energy [cf. Eq. (1)] as a function of the oxygen chemical potential $\mu_{\mathrm{O}}$. We correlate $\mu_{\mathrm{O}}$ with oxygen pressure for different temperatures [cf. Eq. (2)]. The oxygen chemical potential $\mu_{\mathrm{O}}(p, T)$ can, experimentally (and assuming that thermodynamic equilibrium applies), only be varied within certain boundaries. The lower boundary, which will be called the O-lean limit, is defined such that the decomposition of the oxide into cerium metal and molecular oxygen becomes energetically favorable. An appropriate upper boundary for $\mu_{\mathrm{O}}$, on the other hand (the O-rich limit), is given by gas phase conditions that are so rich in oxygen that oxygen condensation will start on the surface at low enough temperatures. Reasonable and well-defined estimates for these limits ${ }^{48}$ are given by 

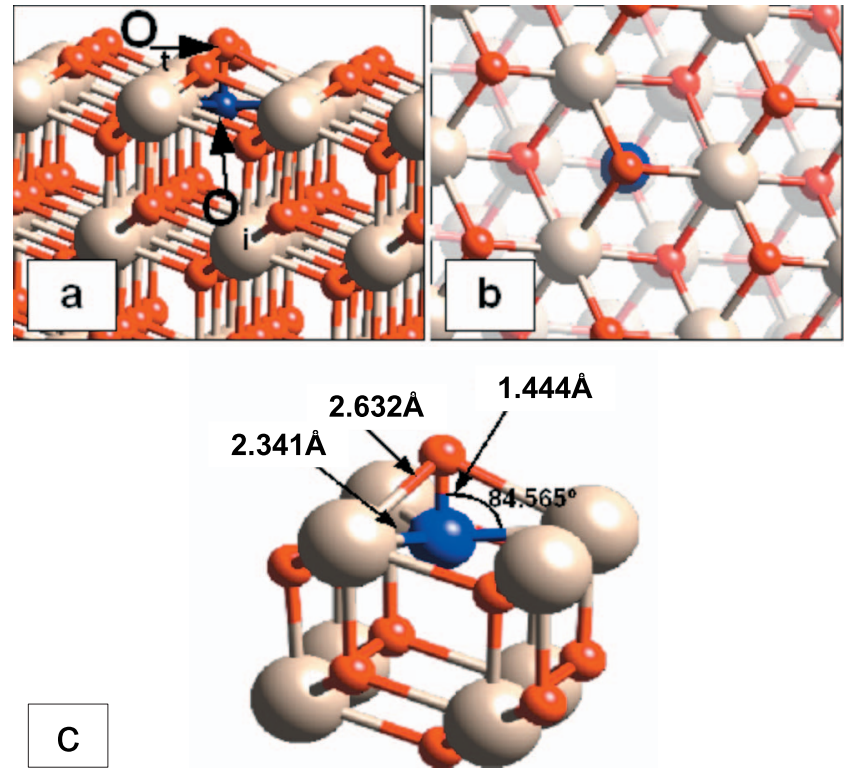

FIG. 14. Side view (a), top view (b), and local atomic geometry (c) of the $\mathrm{CeO}_{2}(111)+\mathrm{O}_{i}$ surface. Various bond lengths and angles are indicated (c). The medium dark (blue) sphere represents the interstitial $\mathrm{O}_{i}$ atoms.

$$
\frac{1}{2} \Delta H_{f}(p=0, T=0 \mathrm{~K})<\Delta \mu_{\mathrm{O}}\left(p_{\mathrm{O}_{2}}, T\right)<0,
$$

where the $\mathrm{O}$ chemical potential is referenced with respect to half the total energy of an oxygen molecule, $\Delta \mu_{\mathrm{O}}=\mu_{\mathrm{O}}$ $-(1 / 2) E_{\mathrm{O}_{2}}^{\text {total }}$, and $\Delta H_{f}(p=0, T=0 \mathrm{~K})$ is the low temperature limit of the heat of formation of $\mathrm{CeO}_{2}$. We calculate $\Delta H_{f}(p$ $=0, T=0 \mathrm{~K})=-10.03 \mathrm{eV}$, thus, $1 / 2 \Delta H_{f}=-5.01 \mathrm{eV}$. In Figs. 15 and 16, which show the surface free energy for the surface structures described above, the value of $1 / 2 \Delta H_{f}$ is indicated by the line "A" (vertical black dashed line) (Table $\mathrm{V})$. It can be seen clearly that the most stable surface for a wide range of the oxygen chemical potential (from -3.4 to $0 \mathrm{eV}$ ) is the stoichiometric $\mathrm{CeO}_{2}(111)$ surface. For higher values of $\Delta \mu_{\mathrm{O}}$, we can also consider the $\mathrm{CeO}_{2}(111)$ $+\mathrm{O}_{i}$ configuration to be a surface of interest, in view of the fact that ceria is well known for its high oxygen storage capacity. For the range of oxygen chemical potential, $-4 \mathrm{eV} \leqslant \Delta \mu_{\mathrm{O}} \leqslant-3.4 \mathrm{eV}$, the most stable structure considered is the $\mathrm{CeO}_{2}(111)$ surface with an oxygen vacancy, $\mathrm{CeO}_{2}(111)+V_{\mathrm{O}}$, while for $\Delta \mu_{\mathrm{O}} \leqslant-4 \mathrm{eV}$, the $\mathrm{CeO}_{2}(111): \mathrm{Ce}$ surface has the lowest energy. A similar trend for the relative stability of ceria surfaces was reported by Jiang et al. in a recent DFT-PBE study using the PAW method. ${ }^{38}$ In particu-

TABLE IV. Surface free energy of $\mathrm{CeO}_{2}$ surfaces for oxygen-lean and oxygen-rich limits (in $\mathrm{eV} / \AA^{2}$ ).

\begin{tabular}{lrc}
\hline \hline & $E_{\text {O-lean }}$ & $E_{\text {O-rich }}$ \\
\hline $\mathrm{CeO}_{2}(111)$ & 0.037 & 0.037 \\
$\mathrm{CeO}_{2}(111): \mathrm{Ce}$ & -0.048 & 0.338 \\
$\mathrm{CeO}_{2}(111): \mathrm{O}$ & 0.668 & 0.281 \\
$\mathrm{CeO}_{2}(110)$ & 0.057 & 0.057 \\
$\mathrm{CeO}_{2}(100): \mathrm{O}$ & 0.565 & 0.230 \\
$\mathrm{CeO}_{2}(100): \mathrm{Ce}$ & 0.008 & 0.343 \\
\hline \hline
\end{tabular}

lar, they found that for $\mathrm{CeO}_{2}$ surfaces, the relative order of stability is as follows:

$$
(111)>(110)>(210)>(211)>(100)>(310) .
$$

From their calculations, at $T=300 \mathrm{~K}$, the stoichiometric (111) surface has the lowest surface energy over almost the entire range of pressures up to $1 \mathrm{~atm}$. With decreasing partial pressures (or for lower $\Delta \mu_{\mathrm{O}}$ ), the Ce-terminated (111) surface becomes more favorable. We note that it is not clear if the Ce-terminated (111) surface depicted in Ref. 38 has the same atomic structure as ours; however, it is evident that with decreasing $\Delta \mu_{\mathrm{O}}$, Ce-rich surfaces appear more stable than the stoichiometric (111) surface. It should also be noted that the study by Jiang et al. only investigated surfaces with ideal terminations, i.e., without the inclusion of defects. Hence, the low-energy $\mathrm{CeO}_{2}(111)+V_{\mathrm{O}}$ structure was not captured in their work for $-4 \mathrm{eV} \leqslant \Delta \mu_{\mathrm{O}} \leqslant-3.4 \mathrm{eV}$, and such surfaces with oxygen vacancies could well be catalytically relevant structures. ${ }^{30,72}$ We note that we also considered a subsurface $\mathrm{O}$ vacancy at the $\mathrm{CeO}_{2}(111)$ surface which we find lower in energy than the on-surface vacancy (in agreement with other groups) by $4 \mathrm{meV} \AA^{2}$, which corresponds to a $0.30 \mathrm{eV}$ lower vacancy formation energy. The values of the $\mathrm{O}$-vacancy formation energies for the surface and subsurface vacancies that we obtain are 3.03 and $2.73 \mathrm{eV}$, respectively. This is similar to those obtained in Ref. 37 where a value of $3.39 \mathrm{eV}$ was reported for the surface $\mathrm{O}$ vacancy, and the subsurface vacancy was $0.18 \mathrm{eV}$ lower. We note that the study used a smaller surface unit cell of $(\sqrt{3} \times 2)$ rectangular in which there are stronger repulsive interactions between the vacancies. In particular, Ref. 37 found using a smaller $(\sqrt{3} \times 1)$ rectangular surface unit cell the value is $3.98 \mathrm{eV}$. Thus, for a larger $(2 \times 2)$ cell as used in our work, the formation energy could be expected to be somewhat less than $3.39 \mathrm{eV}$. We note also that the surface energy for the clean stoichiometric $\mathrm{CeO}_{2}(111)$ surface of $0.039 \mathrm{eV} / \AA^{2}$ of Ref. 37 is in very close agreement with our value of $0.037 \mathrm{eV} / \AA^{2}$. Our findings regarding surface and subsurface oxygen vacancies are also in very good agreement with the findings of Ref. 41 where the reported energy differences are $0.45 \mathrm{eV}$ (hybrid functionals, $\mathrm{HF}), 0.47 \mathrm{eV}(\mathrm{PBE}+U)$, and $0.22 \mathrm{eV}$ $(\mathrm{LDA}+U)$. The values of the $\mathrm{O}$ vacancy formation energies for the surface vacancies are reported in Ref. 41 to be $3.10 \mathrm{eV}(\mathrm{HF}), 3.21 \mathrm{eV}(\mathrm{LDA}+U)$, and $2.34 \mathrm{eV}(\mathrm{GGA}+U)$. In the Appendix we plot these results as surface energies as a function of the oxygen chemical potential.

As mentioned in Sec. III D, the distinct structural resemblance seen for the top layers of the Ce-rich (111) surface, i.e., $\mathrm{CeO}_{2}(111)$ : $\mathrm{Ce}$ and $\mathrm{Ce}_{2} \mathrm{O}_{3}(0001)$ could be indicative of a possible surface phase transition taking place. It can be seen from Fig. 16 that this $\mathrm{CeO}_{2}(111)$ : Ce surface becomes more favorable than the stoichiometric (111) surface under strongly reducing conditions $\left(\Delta \mu_{\mathrm{O}} \leqslant-4 \mathrm{eV}\right)$. In fact, the $\mathrm{Ce}_{2} \mathrm{O}_{3}(0001) / \mathrm{CeO}_{2}(111)$ interface was first investigated theoretically using DFT-LDA by Yamamoto et $a l^{44}$ in an attempt to understand the dielectric properties of this class of material. The authors, however, did not report the energetics of this $\mathrm{Ce}_{2} \mathrm{O}_{3}(0001) / \mathrm{CeO}_{2}(111)$ interface. Thus, our study represents a first investigation of the energetics associated 

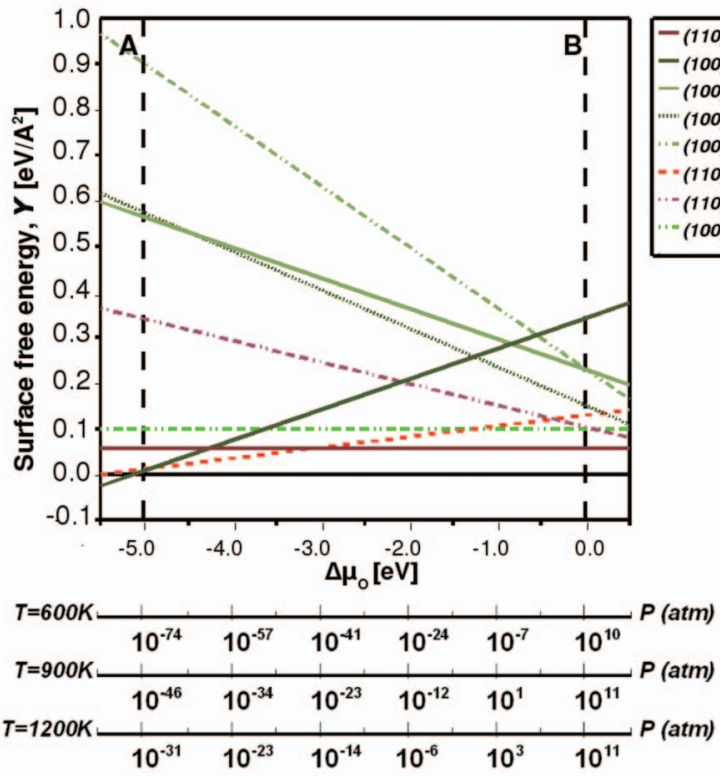

FIG. 15. Calculated surface free energy of various considered $\mathrm{CeO}_{2}(100)$ and $\mathrm{CeO}_{2}(110)$ surfaces as a function of the change in oxygen chemical potential $\Delta \mu_{\mathrm{O}}$ with the corresponding pressure bar lines at $T=600,900$, and $1200 \mathrm{~K}$. "A" and "B" represent the O-lean and O-rich extremes, respectively. Three low-energy structures [the stoichiometric $\mathrm{CeO}_{2}(111)$, $\mathrm{CeO}_{2}(111)+V_{\mathrm{O}}$, and $\left.\mathrm{CeO}_{2}(111): \mathrm{Ce}\right]$ are predicted, depending on the value of $\Delta \mu_{\mathrm{O}}$.

with the initial stages of $\mathrm{CeO}_{2}$ reduction to $\mathrm{Ce}_{2} \mathrm{O}_{3}$ via the (111) surface. Admittedly, we are aware that perhaps our current study could well be impeded by the fact that the electronic structure of $\mathrm{Ce}_{2} \mathrm{O}_{3}$ is poorly described by conventional DFT. However, concerning the fair agreement of the atomic geometry and energetics of this sesquioxide of cerium between DFT, calculations going beyond plain DFT and experiment, we propose that DFT should be able to provide (at least) a qualitative understanding of the energetics of this surface transition.

Da Silva et al. ${ }^{22}$ calculated the heat of reduction for bulk $\mathrm{CeO}_{2}$ to bulk $\mathrm{Ce}_{2} \mathrm{O}_{3}, \Delta H^{2 \mathrm{CeO}_{2}} \rightarrow \Delta H^{\mathrm{Ce}_{2} \mathrm{O}_{3}}$, using difference approaches, namely, DFT, DFT $+U$, and hybrid DFT. The value they obtain from DFT-GGA (PBE) is $-4.18 \mathrm{eV}$. Our calculated value is $-4.11 \mathrm{eV}$ and experimentally reported values are -4.03 (Ref. 73) and $-3.57 \mathrm{eV}^{74}$ It is proposed

TABLE V. Surface free energy of $\mathrm{CeO}_{2}$ surfaces with defects for oxygenlean and oxygen-rich limits (in $\mathrm{eV} / \AA^{2}$ ).

\begin{tabular}{lrc}
\hline \hline & $E_{\text {O-lean }}$ & $E_{\text {O-rich }}$ \\
\hline $\mathrm{CeO}_{2}(111)+V_{\mathrm{O}}$ & 0.006 & 0.103 \\
$\mathrm{CeO}_{2}(111)+V_{\mathrm{O}(\mathrm{s})}$ & -0.001 & 0.096 \\
$\mathrm{CeO}_{2}(111)+V_{\mathrm{Ce}}$ & 0.154 & 0.348 \\
$\mathrm{CeO}_{2}(111): \mathrm{O}+V_{\mathrm{O}}$ & 0.390 & 0.100 \\
$\mathrm{CeO}_{2}(111): \mathrm{O}+V_{\mathrm{Ce}}$ & 0.812 & 0.232 \\
$\mathrm{CeO}_{2}(111)+\mathrm{O}_{i}$ & 0.150 & 0.060 \\
$\mathrm{CeO}_{2}(110)+V_{\mathrm{O}}$ & 0.012 & 0.130 \\
$\mathrm{CeO}_{2}(110)+V_{\mathrm{Ce}}$ & 0.342 & 0.105 \\
$\mathrm{CeO}_{2}(100): \mathrm{O}+V_{\mathrm{O}}$ & 0.575 & 0.152 \\
$\mathrm{CeO}_{2}(100): \mathrm{O}+V_{\mathrm{Ce}}$ & 0.902 & 0.232 \\
$\mathrm{CeO}_{2}(100): \mathrm{Ce}+V_{\mathrm{O}}$ & 0.016 & 0.232 \\
$\mathrm{CeO}_{2}(100): \mathrm{Ce}+V_{\mathrm{Ce}}$ & 0.098 & 0.098 \\
\hline \hline
\end{tabular}
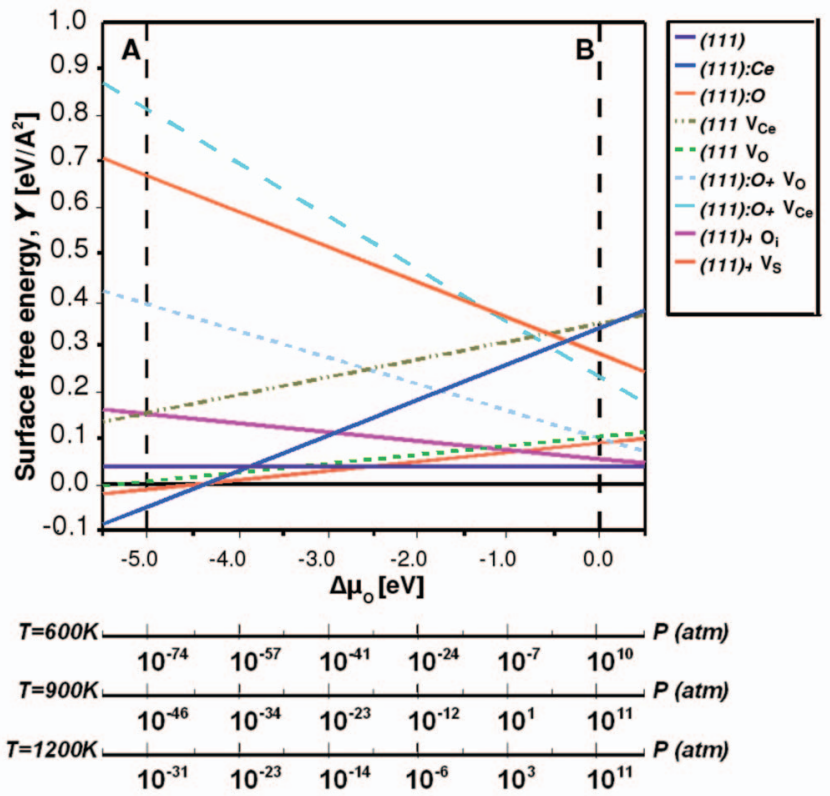

FIG. 16. Calculated surface free energy of various considered $\mathrm{CeO}_{2}(111)$ surfaces as a function of the change in oxygen chemical potential $\Delta \mu_{\mathrm{O}}$ with the corresponding pressure bar lines at $T=600,900$, and $1200 \mathrm{~K}$. "A" and "B" represent the O-lean and O-rich extremes, respectively. Three lowenergy structures [the stoichiometric $\mathrm{CeO}_{2}(111), \mathrm{CeO}_{2}(111)+V_{\mathrm{O}}$, and $\left.\mathrm{CeO}_{2}(111): \mathrm{Ce}\right]$ are predicted, depending on the value of $\Delta \mu_{\mathrm{O}}$.

that DFT systematically underestimates the bulk formation energies, and consequently overestimates the heat of reduction, as is also found for a large class of other oxides. ${ }^{75} \mathrm{In}$ passing, we note that the authors of Ref. 22 found that using the hybrid DFT functionals (which are proposed to improve the exchange-correlation treatment for correlated systems) did not improve the description of the heat of reduction significantly. By simply considering the bulk-phase reduction energy, it could be indicative that a phase transition indeed takes place at very low oxygen chemical potentials $\left(\Delta \mu_{\mathrm{O}} \leqslant-4.11 \mathrm{eV}\right)$, i.e., under highly reducing conditions. This is illustrated in Fig. 17 in which the stability of bulk

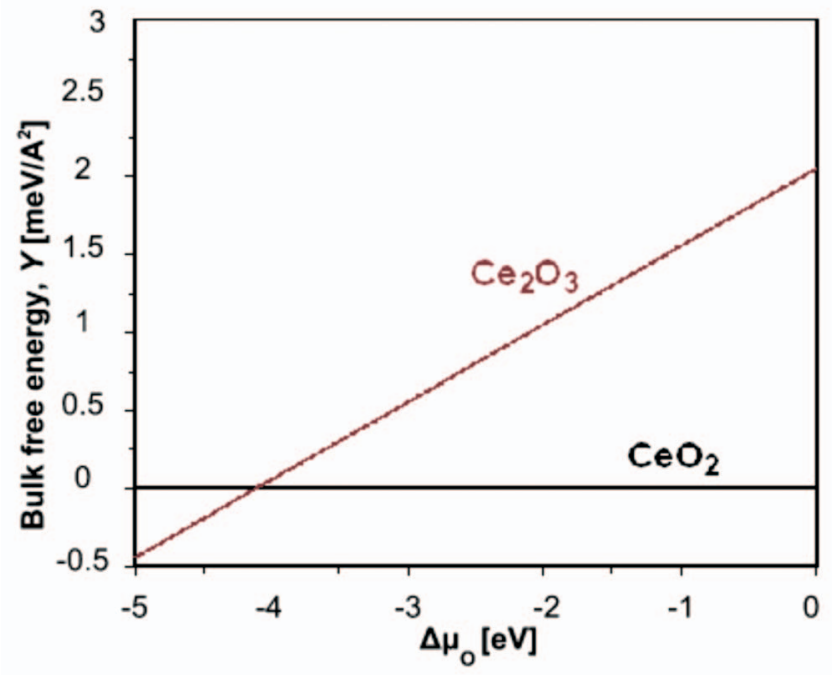

FIG. 17. Relative stability of bulk $\mathrm{Ce}_{2} \mathrm{O}_{3}$ and bulk $\mathrm{CeO}_{2}$ per $\mathrm{Ce}$ atom vs the chemical potential of the oxygen. For $\Delta \mu_{\mathrm{O}} \leqslant-4.11 \mathrm{eV}$, the $\mathrm{Ce}_{2} \mathrm{O}_{3}$ phase is more stable than the $\mathrm{CeO}_{2}$ phase. 


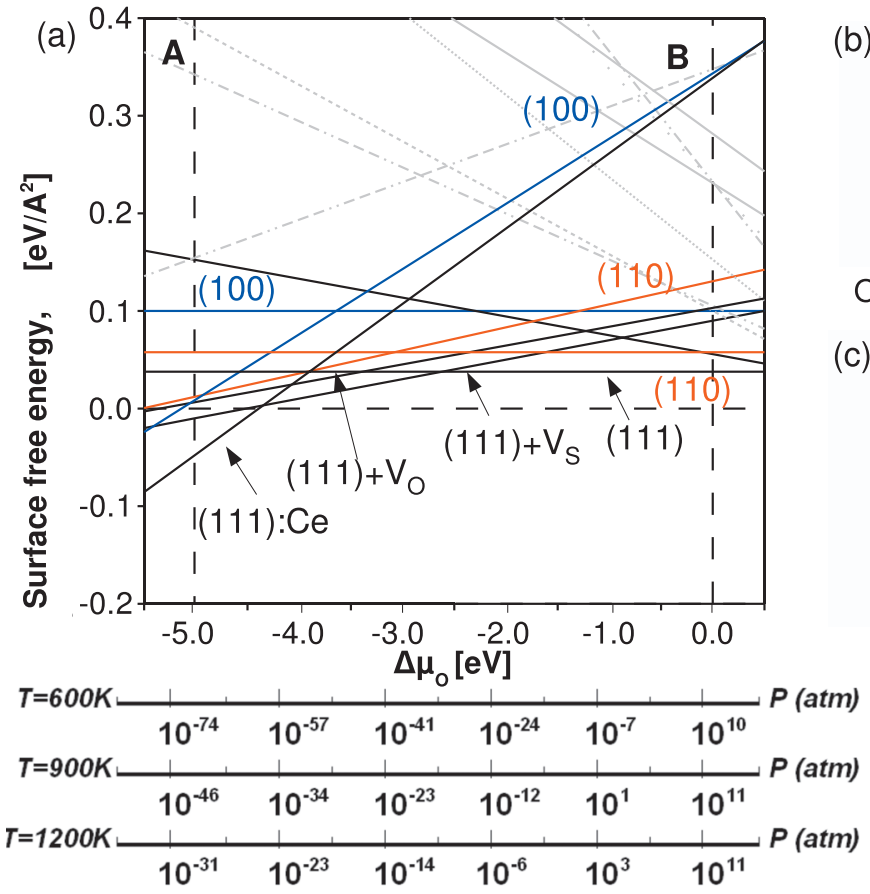

$\mathrm{Ce}_{2} \mathrm{O}_{3}$ is shown relative to bulk $\mathrm{CeO}_{2}$ per $\mathrm{Ce}$ atom versus oxygen chemical potential.

\section{G. Crystal morphology: Dependence on oxygen environment}

Having obtained the surface Gibbs free energies (as a function of the chemical potential of oxygen) of different low-index surface structures, we can then utilize these energies to predict the morphology of $\mathrm{CeO}_{2}$ nanoparticles using the Gibbs-Wulff theorem of equilibrium crystal shape (ECS). ${ }^{76,77}$ As we are only using the surface energies of lowindex surfaces, we are essentially predicting a "constrained" ECS, confined by these low-index surfaces. In fact, it has been shown from experiments ${ }^{78-80}$ that the as-synthesized crystals are typically terminated by low-index surfaces, thus higher-index surfaces may not have a strong influence on the nanoparticle shape.

Briefly, the Gibbs-Wulff theorem ${ }^{76,77}$ provides a simple mathematical construction that predicts the ECS of crystals or nanoparticles, whereby the total free energy of the crystal is at a minimum at a constant volume. The predicted ECS is then termed as a Wulff construction. The mathematical expression for this energy-shape relation is as follows:

$$
r(\boldsymbol{d})=\min _{h k l}\left[\alpha \cdot \gamma\left(\Delta \mu_{\mathrm{O}}\right)\right],
$$

where $r(\boldsymbol{d})$ represents the radius of the crystal shape in the direction of the vector, $\boldsymbol{d}$, and $\alpha$ is the proportionality constant. The directional vector $\boldsymbol{d}$ defines the normal vector to a particular crystal surface $(h k l)$. At a distance from the origin numerically equal to the surface free energy, $\gamma\left(\Delta \mu_{\mathrm{O}}\right)$, of that surface, a normal plane is established. Consequently, one returns to the origin and reiterates this process for all other crystallographic directions. In this way, the smallest volume enveloping the origin inside all these planes can then be taken, in a geometrical sense, as the minimum-energy mor-
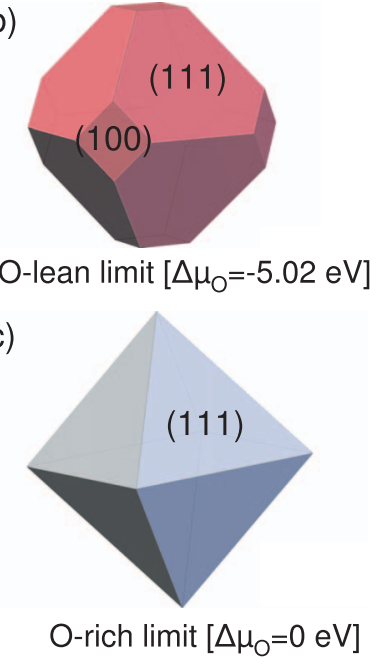

FIG. 18. (a) Enlarged plot of surface energy vs the change in oxygen chemical potential. Only the most favorable surface terminations are labeled while the less favorable ones are shown in pale gray. The predicted crystal morphologies of $\mathrm{CeO}_{2}$ under (b) oxygen-lean and (c) oxygen-rich conditions correspond to $\Delta \mu_{\mathrm{O}}=-5.02$ and $0 \mathrm{eV}$, respectively. The exposed facets are labelled accordingly. phology for a particular crystal. Since the surface free energy can also be expressed as a function of the chemical potential of oxygen $\left(\Delta \mu_{\mathrm{O}}\right)$, this allows a rather straightforward extension of the Wulff construction to include a chemical potential dependence.

Using this approach, the predicted crystal morphology of $\mathrm{CeO}_{2}$ under oxygen-lean [Fig. 18(b)] and oxygen-rich [Fig. 18 (c)] conditions for $\Delta \mu_{O}=-5.02$ and $0 \mathrm{eV}$, respectively is predicted. The exposed facets are labeled accordingly, namely, the (100) and (111) facets under oxygen-lean conditions and the strong dominance of the (111) facet under oxygen-rich conditions. In particular, under oxygen-lean conditions, we find that the morphology of the $\mathrm{CeO}_{2}$ crystal is described as a truncated octahedron, dominated by the (111) facet at the faces of the octahedron with the minority (100) facet terminating the apices of the octahedron. This can be inferred from the relative stability of the surface energies of the (111) and (100) surface terminations, namely, that of $\mathrm{CeO}_{2}(111)+V_{\mathrm{O}}$ and $\mathrm{CeO}_{2}(111): \mathrm{Ce}$, respectively, from Fig. 18(a). We note, however, that we have considered the lowenergy (111) surface to be $\mathrm{CeO}_{2}(111)+V_{\mathrm{O}}$ rather than the $\mathrm{CeO}_{2}(111)$ : $\mathrm{Ce}$ as the latter surface structure has a negative surface energy (as discussed above) which is not defined in the Wulff theorem.

Upon moving to higher values of the oxygen chemical potential, the morphology changes slightly to an octahedron shape only exposing only the (111) facet at the eight faces. This is, again, clearly reflected in the relative stability of surface energies in Fig. 18(a), particularly from $\Delta \mu_{\mathrm{O}}=-3.4$ to $0 \mathrm{eV}$. For these range of $\Delta \mu_{\mathrm{O}}$, the low-energy (100), (110), and (111) facets are $\mathrm{CeO}_{2}(100)+V_{\mathrm{Ce}}$, $\mathrm{CeO}_{2}(110)$, and $\mathrm{CeO}_{2}(111)$, respectively, with the stoichiometric $\mathrm{CeO}_{2}(111)$ having the lowest surface energy.

Our predictions are in very good agreement with experimental observations. ${ }^{78-80}$ Recently, the morphology of twodimensional nanostructures of $\mathrm{CeO}_{2}$ was examined using a 
dynamic scanning force microscope and it was found that the surface shows hexagonal islands, exposing the (111) surface on the flat terrace with the (100) and (110) surfaces as step edges. ${ }^{78}$ The authors conclude that under conditions of thermal equilibrium, triangular islands are to be expected [i.e., exposing only the (111) surface], hence suggesting that these observed higher energy step edges are formed mainly due to kinetic hindrances. For three-dimensional structures, Yan et $a{ }^{79}{ }^{79}$ synthesized single-nanocrystalline $\mathrm{CeO}_{2}$ structures using a hydrothermal synthesis process, and by tuning the treatment time, they find the morphology can be controlled and tuned, ranging from nano-octahedrons to nanorods. Also, using a different technique of combustion chemical vapor condensation, Barnard et al. ${ }^{80}$ find nanosized $\mathrm{CeO}_{2}$ particles (from 4 to $6 \mathrm{~nm}$ in size) exhibit distinct extended (111) facets with small (approximately four atomic column wide) (100) facet at the apices, i.e., yielding some sort of truncated octahedron. Admittedly, these experimental conditions may not directly correspond to the simple oxygen pressuretemperature relation we refer to in Fig. 18(a), e.g., conditions may differ in the presence of surfactants and solvents. However, collectively, these results seem to suggest that the predominant morphology of $\mathrm{CeO}_{2}$ crystals is, at least, qualitatively captured by our predicted Wulff construction.

\section{SUMMARY AND CONCLUSION}

We performed DFT calculations for the low-index surfaces of cerium oxide, $\mathrm{CeO}_{2}$, for various terminations and surface defects. The surface free energies were calculated as a function of the oxygen chemical potential, from which we found that the most stable surfaces for decreasing values of the chemical potential (i.e., for increasingly oxygen-lean conditions) are the stoichiometric $\mathrm{CeO}_{2}(111)$ surface, the $\mathrm{CeO}_{2}(111)$ surface with subsurface oxygen vacancies, and the $\mathrm{CeO}_{2}(111)$ : Ce terminated surface. We also find that the $\mathrm{CeO}_{2}(111)$ surface with an interstitial oxygen has a rather low surface free energy under highly oxidizing conditions. Using DFT-GGA, $\mathrm{CeO}_{2}(111)$ is found to be semiconducting, similar to the bulk material, while the same surface with a surface oxygen vacancy is found to be metallic. This metallic behavior is also found for the $\mathrm{Ce}$ terminated $\mathrm{CeO}_{2}(111)$ structure and the $\mathrm{Ce}_{2} \mathrm{O}_{3}(0001)$ surface. This could well be an artifact of DFT-GGA not being able to correct for the unphysical self-interaction of the electrons. Interestingly, from an analysis of the energetics, and the atomic and electronic structure, our results show an indication of a morphological reconstruction of the $\mathrm{CeO}_{2}(111): \mathrm{Ce}$ surface into a $\mathrm{Ce}_{2} \mathrm{O}_{3}(0001)$-like surface, which could occur for highly reducing conditions and possibly represent the initial stages of a phase transformation from $\mathrm{CeO}_{2}$ to $\mathrm{Ce}_{2} \mathrm{O}_{3}$.

\section{ACKNOWLEDGMENTS}

The authors gratefully acknowledge support from the Australian Research Council (ARC), the Australian National Supercomputing Facility, the Australian Partnership for Advanced Computing (APAC), and the Australian Centre for Advanced Computing and Communications (ac3).

\section{APPENDIX: CONVERGENCE TESTS}

For bulk $\mathrm{CeO}_{2}$ we performed convergence tests for the real-space cutoff (R-cut) and the k-point set as shown in Fig. 19. It can be seen from Fig. 19(a) that convergence to within $\leqslant 0.05 \mathrm{meV}$ is obtained for a k-point sampling in the BZ of $8 \times 8 \times 8$ (yielding $35 \mathbf{k}$-points in the IBZ) compared to the $12 \times 12 \times 12 \mathbf{k}$-point set. With regard to the cutoff radius of 11 bohrs, employed in all our calculations, Fig. 19(b) shows that compared to an R-cut of 12 bohrs, the total energy is converged to $\leqslant 1 \mathrm{meV}$.

To test the convergence of our calculations with respect to slab thickness, we use supercells containing symmetric slabs of various thicknesses. In particular, for the (111), (110), and (100) surfaces we use 15, 11, and 13 atomic layers, respectively. Then, by adding one layer to each of these surfaces, we found that the corresponding surface energies change by $\leqslant 2 \mathrm{meV} / \AA^{2}$. We also carried out various convergence tests to determine the optimal number of atomic layers to relax for each low-index surface. In particular, Fig. 20 shows the results for the relaxation of the outermost atomic layers for the stoichiometric $\mathrm{CeO}_{2}(111), \mathrm{CeO}_{2}(110)$, and $\mathrm{CeO}_{2}(100)$ :O surfaces. The optimal value found for the number of layers to relax is two trilayers, three atomic layers, and two atomic layers for the (111), (110), and (100) surfaces, respectively, which ensure an error in the surface energies of $\leqslant 1 \mathrm{meV} / \AA^{2}$.
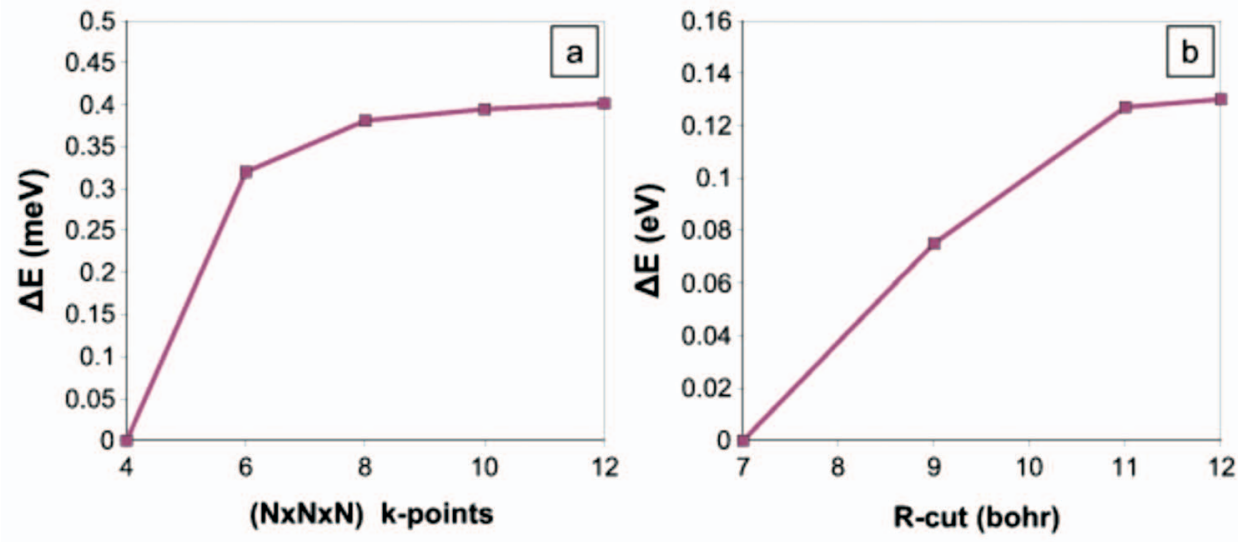

FIG. 19. Change in the total energy vs (a) increasing k-point mesh and (b) cutoff radius for bulk $\mathrm{CeO}_{2}$. 

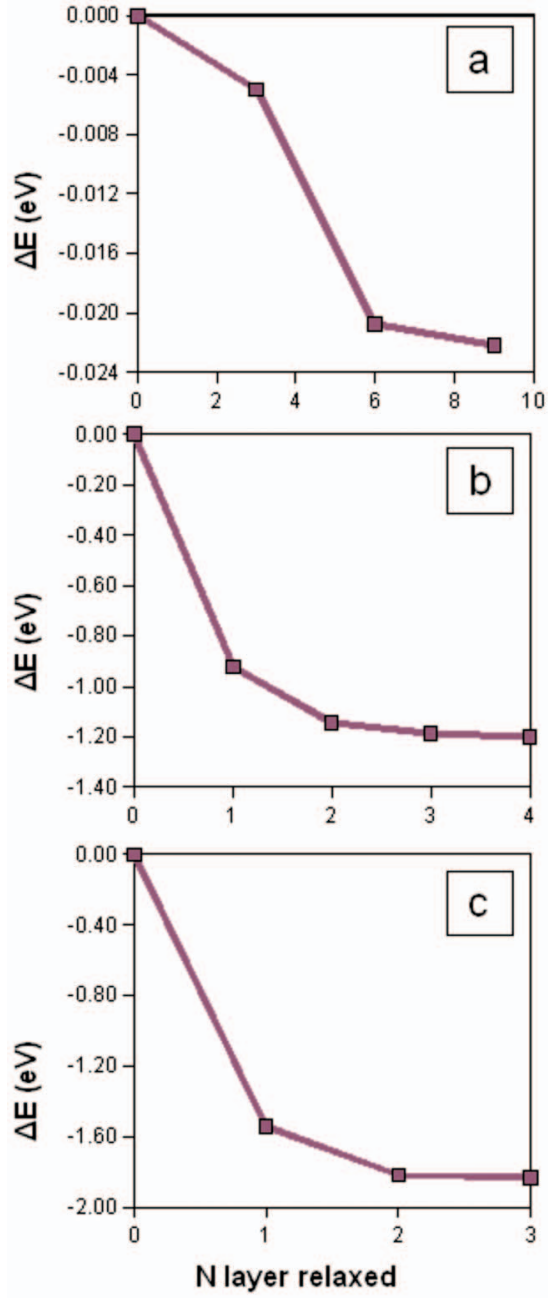

FIG. 20. Change in the total energy vs the number of atomic layers relaxed for (a) $\mathrm{CeO}_{2}(111)$, (b) $\mathrm{CeO}_{2}(110)$, and (c) $\mathrm{CeO}_{2}(100): \mathrm{O}$.

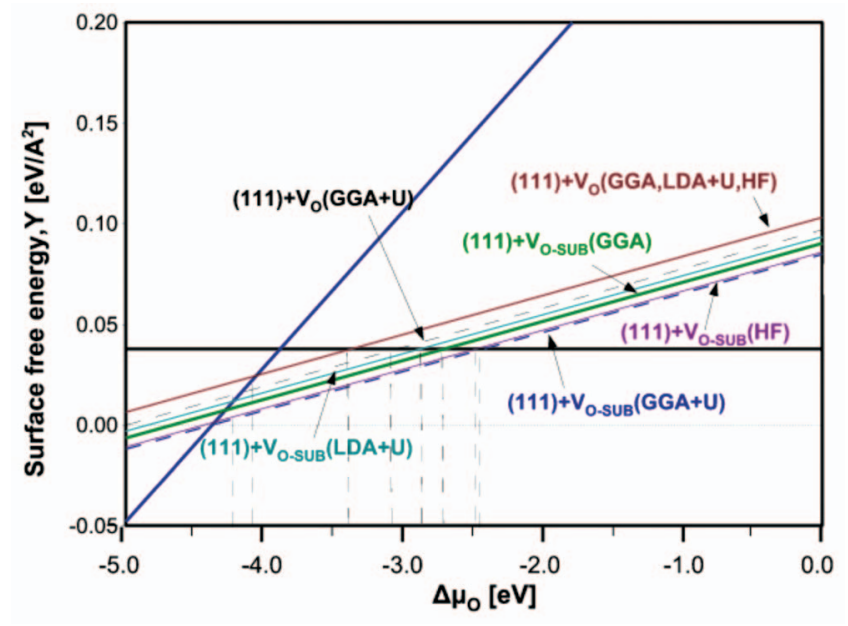

FIG. 21. Surface free energy of $\mathrm{CeO}_{2}(111)$ surfaces with surface and subsurface oxygen vacancies in a $(2 \times 2)$ periodicity, referred to the surface energy of the clean surfaces, as a function of the oxygen chemical potential. The label "(111) $+V_{\mathrm{O}}(\mathrm{GGA}, \mathrm{LDA}+U, \mathrm{HF})$ " corresponds to the present DFT-GGA result, and the DFT-LDA $+U$ and HF results of Ref. 41 for the surface oxygen vacancy (the lines lie practically on top of each other). The label " $(111)+V_{\mathrm{O}}(\mathrm{GGA}+U) "$ is the result obtained using the DFT-GGA(PBE) $+U$ approach of Ref. 41. The subscript "O-SUB" is the result for the subsurface oxygen vacancy. All " $+U$ " and "HF" results are from Ref. 41.
In Fig. 21 we plot the surface free energy of the stoichiometric $\mathrm{CeO}_{2}$ (111) surface and that of the surface with surface and subsurface vacancies, as obtained in the present work, in comparison with the recent results of Ref. 41, which highlight the consistency of our results with the DFT $+U$ and HF approaches.

${ }^{1}$ M. S. Dresselhaus and I. L. Thomas, Nature (London) 414, 332 (2001).

${ }^{2}$ V. Esposito and E. Traversa, J. Am. Ceram. Soc. 91, 1037 (2008).

${ }^{3}$ L. Saraf, C. M. Wang, V. Shutthanandan, Y. Zhang, O. Marina, D. Baer, S. Thevuthasan, P. Nachimuthu, and D. Lindle, J. Mater. Res. 20, 1295 (2005).

${ }^{4}$ S. Kim and J. Maier, J. Eur. Ceram. Soc. 24, 1919 (2004).

${ }^{5}$ A. Trovarelli, Catalytic Science 2, 407 (2002).

${ }^{6}$ A. Trovarelli, Catal. Rev. - Sci. Eng. 38, 439 (1996).

${ }^{7}$ M. Yano, A. Tomita, M. Sano, and T. Hibino, Solid State Ionics 177, 3351 (2007)

${ }^{8}$ T. Hibino, A. Hashimoto, T. Inoue, J. Tokuno, S. Yoshida, and M. Sano, Science 288, 231 (2000).

${ }^{9}$ T. Hibino, A. Hashimoto, T. Inoue, S. Yoshiba, and M. Sano, J. Electrochem. Soc. 147, 2888 (2000).

${ }^{10}$ A. V. Prokofiev, A. I. Shelykh, and B. T. Melekh, J. Alloys Compd. 242, 41 (1996)

${ }^{11}$ V. I. Anisimov, J. Zaanen, and O. K. Andersen, Phys. Rev. B 44, 943 (1991).

${ }^{12}$ I. V. Solovyev, P. H. Dederichs, and V. I. Anisimov, Phys. Rev. B 50, 16861 (1994).

${ }^{13}$ M. Cococcioni and S. De Gironcoli, Phys. Rev. B 71, 035105 (2005).

${ }^{14}$ M. V. Ganduglia-Pirovano, A. Hofmann, and J. Sauer, Surf. Sci. Rep. 62, 219 (2007).

${ }^{15}$ C. Loschen, J. Carrasco, K. M. Neyman, and F. Illas, Phys. Rev. B 75, 035115 (2007).

${ }^{16}$ J. L. F. Da Silva, Phys. Rev. B 76, 193108 (2007).

${ }^{17}$ D. A. Andersson, S. I. Simak, B. Johansson, I. A. Abrikosov, and N. V. Skorodumova, Phys. Rev. B 75, 035109 (2007).

${ }^{18}$ G. Pacchioni, J. Chem. Phys. 128, 182505 (2008).

${ }^{19}$ C. W. M. Castleton, J. Kullgren, and K. Hermansson, J. Chem. Phys. 127, 244704 (2007).

${ }^{20}$ P. J. Hay, R. L. Martin, J. Uddin, and G. E. Scuseria, J. Chem. Phys. 125, 034712 (2006).

${ }^{21}$ E. Voloshina and B. Paulus, J. Chem. Phys. 124, 234711 (2006).

${ }^{22}$ J. L. F. Da Silva, M. V. Ganduglia-Pirovano, and J. Sauer, Phys. Rev. B 75, 045121 (2007).

${ }^{23}$ A. Pfau and K. D. Schierbaum, Surf. Sci. 321, 71 (1994).

${ }^{24}$ E. Wuilloud, B. Delley, W. D. Schneider, and Y. Baer, Phys. Rev. Lett. 53, 202 (1984).

${ }^{25}$ D. R. Mullins, S. H. Overbury, and D. R. Huntley, Surf. Sci. 409, 307 (1998).

${ }^{26}$ J. P. Holgado, R. Alvarez, and G. Munuera, Appl. Surf. Sci. 161, 301 (2000).

${ }^{27}$ S. Wang, Z. Qiao, and Y. Q. Wei Wang, J. Alloys Compd. 305, 121 (2000).

${ }^{28}$ L. A. J. Garvie and P. R. Buseck, J. Phys. Chem. Solids 60, 1943 (1999).

${ }^{29}$ A. Fujimori, Phys. Rev. Lett. 53, 2518 (1984).

${ }^{30}$ F. Esch, S. Fabris, L. Zhou, T. Montini, C. Africh, P. Fornasiero, G. Comelli, and R. Rosei, Science 309, 752 (2005).

${ }^{31}$ T. X. T. Sayle, S. Parker, C. Richard, and A. Catlow, J. Phys. Chem. 98, 13625 (1994).

${ }^{32}$ J. C. Conesa, Surf. Sci. 339, 337 (1995)

${ }^{33}$ S. E. Hill, C. Richard, and A. Catlow, J. Phys. Chem. Solids 54, 411 (1992).

${ }^{34}$ S. Gennard, F. Cora, C. Richard, and A. Catlow, J. Phys. Chem. 103, 10158 (1999).

${ }^{35}$ N. V. Skorodumova, R. Ahuja, S. I. Simak, I. A. Abrikosov, B. Johansson, and B. I. Lundqvist, Phys. Rev. B 64, 115108 (2001).

${ }^{36}$ N. V. Skorodumova, M. Baudin, and K. Hermansson, Phys. Rev. B 69, 075401 (2004).

${ }^{37}$ Z. Yang, T. K. Woo, M. Baudin, and K. Hermansson, J. Chem. Phys. 120, 7741 (2004)

${ }^{38}$ Y. Jiang, J. B. Adams, and M. van Schilfgaarde, J. Chem. Phys. 123, 064701 (2005).

${ }^{39}$ S. Fabris, S. De Gironcoli, S. Baroni, G. Vicario, and G. Balducci, Phys. Rev. B 71, 041102 (2005). 
${ }^{40}$ S. Fabris, G. Vicario, G. Balducci, S. De Gironcoli, and S. Baroni, J. Phys. Chem. B 109, 22860 (2005).

${ }^{41}$ M. V. Ganduglia-Pirovano, J. L. F. Da Silva, and J. Sauer, Phys. Rev. Lett. 102, 026101 (2009).

${ }^{42}$ H.-Y. Li, H.-F. Wang, X.-Q. Gong, Y.-L. Guo, Y. Guo, G. Lu, and P. Hu, Phys. Rev. B 79, 193401 (2009).

${ }^{43}$ M. Huang and S. Fabris, J. Phys. Chem. C 112, 8643 (2008).

${ }^{44}$ T. Yamamoto, H. Momida, and T. Hamada, Thin Solid Films 486, 136 (2005).

${ }^{45}$ J. P. Perdew, K. Burke, and M. Erzerhof, Phys. Rev. Lett. 77, 3865 (1996).

${ }^{46}$ B. Delley, J. Chem. Phys. 92, 508 (1990).

${ }^{47}$ B. Delley, J. Chem. Phys. 113, 7756 (2000).

${ }^{48}$ K. Reuter and M. Scheffler, Phys. Rev. B 65, 035406 (2002).

${ }^{49}$ C. Stampfl, M. V. Ganduglia-Pirovano, K. Reuter, and M. Scheffler, Surf. Sci. 500, 368 (2002).

${ }^{50}$ K. Reuter and M. Scheffler, Phys. Rev. B 68, 045407 (2003).

${ }^{51}$ C. Stampfl, Catal. Today 105, 17 (2005).

${ }^{52}$ W. X. Li, C. Stampfl, and M. Scheffler, Phys. Rev. B 68, 165412 (2003).

${ }^{53}$ D. R. Stull and H. Prophet, JANAF Thermochemical Tables, 2nd ed. (U.S. National Bureau of Standards, Washington, D.C., 1971).

${ }^{54}$ C. Kittel, Introduction to Solid State Physics (Wiley, New York, 1996).

${ }^{55}$ A. Soon, M. Todorova, B. Delley, and C. Stampfl, Phys. Rev. B 75 125420 (2007).

${ }^{56}$ B. Johansson, I. A. Abrikosov, and M. Alden, Phys. Rev. Lett. 74, 12 (1995).

${ }^{57}$ P. Soderlin, O. Erikson, and B. Johansson, Phys. Rev. B 50, 7291 (1994).

${ }^{58}$ J. Donohue, The Structure of Elements (Wiley, New York, 1974).

${ }^{59}$ U. Benedict and J. C. Peterson, J. Magn. Magn. Mater. 29, 287 (1982).

${ }^{60}$ B. Amadon, S. Biermann, A. Georges, and F. Aryasetiawan, Phys. Rev. Lett. 96, 066402 (2006).
${ }^{61}$ B. Amadon, F. Jollet, and M. Torrent, Phys. Rev. B 77, 155104 (2008).

${ }^{62}$ J.-P. Rueff, J.-P. Itié, M. Taguchi, C. F. Hague, J.-M. Mariot, R. Delaunay, J.-P. Kappler, and N. Jaouen, Phys. Rev. Lett. 96, 237403 (2006).

${ }^{63}$ Y. Xu and M. Mavrikakis, Surf. Sci. 494, 131 (2001).

${ }^{64}$ W. X. Li, C. Stampfl, and M. Scheffler, Phys. Rev. B 65, 075407 (2002).

${ }^{65} \mathrm{~K}$. Huber and G. Herzberg, Molecular Spectra and Molecular Structure IV: Constant of Diatomic Molecules (Van Nostrand-Reinhold, New York, 1979).

${ }^{66}$ J. Heyd, G. E. Scuseria, and M. Ernzerhof, J. Chem. Phys. 118, 8207 (2003).

${ }^{67}$ C. Adamo and V. Barone, J. Chem. Phys. 110, 6158 (1999).

${ }^{68}$ J. Tao, J. P. Perdew, V. N. Staroverov, and G. E. Scuseria, Phys. Rev. Lett. 91, 146401 (2003).

${ }^{69}$ A. I. Shelykh, A. V. Prokofiev, and B. T. Melekh, Fiz. Tverd. Tela (St. Petersburg) 38, 427 (1996).

${ }^{70}$ D. C. Koskimaki, K. A. Gschneidner, and N. T. Panousis, J. Cryst. Growth 22, 225 (1974).

${ }^{71}$ P. W. Tasker, Solid State Phys. 12, 4977 (1979)

${ }^{72}$ S. Torbrügge, M. Reichling, A. Ishiyama, S. Morita, and Ó. Custance, Phys. Rev. Lett. 99, 056101 (2007).

${ }^{73}$ M. Zinkevich, D. Djurovic, and F. Aldinger, Solid State Ionics 177, 989 (2006).

${ }^{74}$ A. Trovarelli, Catalysis by Ceria and Related Materials (World Scientific, Singapore, 2002).

${ }^{75}$ L. Wang, T. Maxisch, and G. Ceder, Phys. Rev. B 73, 195107 (2006).

${ }^{76}$ G. Wulff, Z. Kristallogr. 34, 449 (1901).

${ }^{77}$ C. Herring, Phys. Rev. 82, 87 (1951).

${ }^{78}$ S. Torbrügge, M. Cranney, and M. Reichling, Appl. Phys. Lett. 93, 073112 (2008).

${ }^{79}$ L. Yan, R. Yu, J. Chen, and X. Xing, Cryst. Growth Des. 8, 1474 (2008).

${ }^{80}$ A. S. Barnard and A. I. Kirkland, Chem. Mater. 20, 5460 (2008). 